TRANSACTIONS OF THE

AMERICAN MATHEMATICAL SOCIETY

Volume 365, Number 8, August 2013, Pages 3943-3970

S 0002-9947(2013)05922-3

Article electronically published on April 24, 2013

\title{
COMPACT COMPOSITION OPERATORS ON BERGMAN-ORLICZ SPACES
}

\author{
PASCAL LEFÈVRE, DANIEL LI, HERVÉ QUEFFÉLEC, AND LUIS RODRÍGUEZ-PIAZZA
}

\begin{abstract}
We construct an analytic self-map $\varphi$ of the unit disk and an Orlicz function $\Psi$ for which the composition operator of symbol $\varphi$ is compact on the Hardy-Orlicz space $H^{\Psi}$, but not on the Bergman-Orlicz space $\mathfrak{B}^{\Psi}$. For that, we first prove a Carleson embedding theorem and then characterize the compactness of composition operators on Bergman-Orlicz spaces, in terms of Carleson function (of order 2). We show that this Carleson function is equivalent to the Nevanlinna counting function of order 2 .
\end{abstract}

\section{INTRODUCTION AND NOTATION}

1.1. Introduction. Due to the Littlewood subordination principle, the boundedness of composition operators $C_{\varphi}$, defined by $C_{\varphi}(f)=f \circ \varphi$, on Hardy spaces $H^{p}$, as well as on Bergman spaces $\mathfrak{B}^{p}, 1 \leq p \leq \infty$, is automatic. Their compactness is something much more subtle, but is well understood now, and there are two well-separated cases. First, the case $p=\infty$, for which $C_{\varphi}: H^{\infty} \rightarrow H^{\infty}$ is compact if and only if $\|\varphi\|_{\infty}<1$ (note that $\mathfrak{B}^{\infty}=H^{\infty}$ ). Second, the case $p<\infty$, for which the compactness does not depend on $p$. For Hardy spaces, this fact, proved by J. Shapiro and P. Taylor ([19]), is not completely trivial and is due to the good factorization properties of functions in $H^{p}$. For the scale of Bergman spaces $\mathfrak{B}^{p}$, the factorization properties are not so good, but the independence with respect to $p$ follows from the characterization ([14], Corollary 4.4): for $1 \leq p<\infty$, $C_{\varphi}: \mathfrak{B}^{p} \rightarrow \mathfrak{B}^{p}$ is compact if and only if the pull-back measure of the area-measure $\mathcal{A}$ by $\varphi$ is a vanishing 2-Carleson measure. The case $p=2$ (proved in [1]) gives then, for $1 \leq p<\infty$,

$$
C_{\varphi}: \mathfrak{B}^{p} \rightarrow \mathfrak{B}^{p} \text { is compact } \Longleftrightarrow \lim _{|z| \rightarrow 1} \frac{1-|\varphi(z)|}{1-|z|}=\infty .
$$

In both cases (Hardy and Bergman), a brutal change of situation occurs when we pass from finite values of $p$ to the value $p=\infty$, and the need was felt for an intermediate scale between $H^{p}$ and $H^{\infty}$, or between $\mathfrak{B}^{p}$ and $\mathfrak{B}^{\infty}$. This is what we did ([7), in full detail, with Hardy-Orlicz spaces $H^{\Psi}$ associated with an Orlicz function $\Psi$ (and began to do for Bergman-Orlicz spaces $\mathfrak{B}^{\Psi}$ ). We introduced a generalization of the notion of Carleson measure and proved a contractivity property of those Carleson measures $m_{\varphi}=\varphi^{*}(m)$, attached to an analytic self-map $\varphi: \mathbb{D} \rightarrow \mathbb{D}$,

Received by the editors May 4, 2010.

2010 Mathematics Subject Classification. Primary 47B33; Secondary 30J10, 30H10, 30J99, $46 \mathrm{E} 15$.

Key words and phrases. Bergman-Orlicz space, Carleson function, compactness, composition operator, Hardy-Orlicz space, Nevanlinna counting function.

(C) 2013 American Mathematical Society Reverts to public domain 28 years from publication 
which turned out to be central to obtaining a necessary and sufficient condition for the compactness of $C_{\varphi}: H^{\Psi} \rightarrow H^{\Psi}$. In that paper, we also began a study of the compactness of composition operators on $\mathfrak{B}^{\Psi}$. We proved, in particular, but implicitly (see the comments at the beginning of Section 4), that, if the Orlicz function $\Psi$ grows very fast (satisfying the so-called $\Delta^{2}$ condition), then the compactness of $C_{\varphi}: H^{\Psi} \rightarrow H^{\Psi}$ implies its compactness as an operator $C_{\varphi}: \mathfrak{B}^{\Psi} \rightarrow \mathfrak{B}^{\Psi}$. On the other hand, it is well known that the compactness on $H^{p}$ implies the compactness on $\mathfrak{B}^{p}$ because it is easy to see that the right-hand side of (1.1) is implied by the compactness on $H^{p}$. One might think that it is generally easier to achieve compactness on $\mathfrak{B}^{\Psi}$ than on $H^{\Psi}$. The main result of the present work is the existence of an analytic self-map $\varphi$ of $\mathbb{D}$ and an Orlicz function $\Psi$ such that the composition operator $C_{\varphi}$ is compact on $H^{\Psi}$ but not on $\mathfrak{B}^{\Psi}$. For that, we first have to characterize the compactness of composition operators on Bergman-Orlicz spaces. More precisely, the paper is organized as follows.

In Section 2, given two Orlicz functions $\Psi_{1}$ and $\Psi_{2}$, and a finite positive measure $\mu$ on the unit disk $\mathbb{D}$, we investigate under which conditions the canonical inclusion $I_{\mu}: \mathfrak{B}^{\Psi_{1}} \rightarrow L^{\Psi_{2}}(\mu)$, defined by $I_{\mu}(f)=f$, is either bounded or compact. In Theorem 2.1. we give a necessary condition and a sufficient condition, in terms of the Carleson function $\rho_{\mu}$ of $\mu$, for the boundedness of $I_{\mu}$. Analoguously, we have a similar statement (Theorem 2.5) for the compactness of $I_{\mu}$. In general, these necessary and those sufficient conditions do not fit.

In Section 3 we prove one of the main results of this paper (Theorem 3.1) under the form of a contractivity principle for the pull-back measure $\mathcal{A}_{\varphi}$ of the planar Lebesgue measure $\mathcal{A}$ on $\mathbb{D}$ by $\varphi$. The proof is rather long and uses a CalderónZygmund decomposition, as well as an elementary, but very useful, inequality due to Paley and Zygmund. This contractivity principle eliminates the absence of fitness mentioned above and allows us to have a necessary and sufficient condition for the compactness of $C_{\varphi}: \mathfrak{B}^{\Psi} \rightarrow \mathfrak{B}^{\Psi}$ in terms of the same Carleson function $\rho_{\mathcal{A}_{\varphi}}=\rho_{\varphi, 2}$ (Theorem 3.2).

In Subsection 3.2. we consider the Nevanlinna counting function $N_{\varphi, 2}$ (initiated in [18]), adapted to the Bergman case, and we compare it with the 2-Carleson function $\rho_{\varphi, 2}$ of $\varphi$. These two functions turn out to be equivalent, in the sense made precise in Theorem 3.10 . This extends to the Bergman case and (follows from) such an equivalence for the Hardy case, that we recently established in [9], Theorem 1.1.

Finally, in Section 4, we exploit the necessary and sufficient conditions that we established, either on $H^{\Psi}$ or on $\mathfrak{B}^{\Psi}$, to give (Theorem 4.2) an example of an analytic self-map $\varphi: \mathbb{D} \rightarrow \mathbb{D}$ and of a fairly irregularly varying Orlicz function $\Psi$ such that, contrary to the general intuition, $C_{\varphi}: H^{\Psi} \rightarrow H^{\Psi}$ is compact, whereas $C_{\varphi}: \mathfrak{B}^{\Psi} \rightarrow \mathfrak{B}^{\Psi}$ is not compact. This is due to the fact that we can evaluate, in an accurate way, the two Carleson functions $\rho_{\varphi}$ and $\rho_{\varphi, 2}$ of $\varphi$.

1.2. Notation. We shall denote by $\mathbb{D}$ the open unit disk $\{z \in \mathbb{C} ;|z|<1\}$ of the complex plane, and its boundary, the unit circle, by $\mathbb{T}$. The normalized area measure $d \mathcal{A}=d x d y / \pi$ on $\mathbb{D}$ will be denoted by $\mathcal{A}$.

For any $\xi \in \mathbb{T}$, we define, for $0<h<1$, the Carleson window $W(\xi, h)$ by

$$
W(\xi, h)=\{z \in \mathbb{D} ;|z| \geq 1-h \quad \text { and } \quad|\arg (z \bar{\xi})| \leq \pi h\} .
$$


We shall also use the "circular" Carleson windows $S(\xi, h)$ defined by $S(\xi, h)=\{z \in$ $\mathbb{D} ;|z-\xi|<h\}$. Since $S(\xi, h) \subseteq W(\xi, h) \subseteq S(\xi, 5 h)$, the measures of $W(\xi, h)$ and of $S(\xi, h)$ are equivalent, up to constants.

For any finite positive measure $\mu$ on $\mathbb{D}$, we define, for $0<h \leq 1$, the Carleson function of $\mu$ by

$$
\rho_{\mu}(h)=\sup _{|\xi|=1} \mu(W(\xi, h))
$$

and we set

$$
K_{\mu, 2}(h)=\sup _{0<t<h} \frac{\rho_{\mu}(t)}{t^{2}} .
$$

When $\rho_{\mu}(h)=O\left(h^{2}\right)$, one says that $\mu$ is a 2-Carleson measure; we also say that $\mu$ is a Bergman-Carleson measure, to insist that the order 2 is adapted to the Bergman spaces. When $\mu=\mathcal{A}_{\varphi}$ is the pull-back measure of $\mathcal{A}$ by an analytic self-map $\varphi: \mathbb{D} \rightarrow \mathbb{D}$, we shall simply write $\rho_{\varphi, 2}$ and $K_{\varphi, 2}$ instead of $\rho_{\mathcal{A}_{\varphi}}$ and $K_{\mathcal{A}_{\varphi}, 2}$, respectively. We shall say that $\rho_{\varphi, 2}$ is the 2-dimensional Carleson function of $\varphi$.

The Hastings-Luecking sets of size $2^{-n}$ are defined by

$$
\Delta_{k}=\left\{z \in \mathbb{D} ; 1-\frac{1}{2^{n}} \leq|z|<1-\frac{1}{2^{n+1}} \quad \text { and } \quad \frac{(2 j-1) \pi}{2^{n}} \leq \arg z<\frac{(2 j+1) \pi}{2^{n}}\right\},
$$

where $k=2^{n}+j-1, n \geq 0,0 \leq j \leq 2^{n}-1$ (note that $\Delta_{0}=D(0,1 / 2)$ ).

An Orlicz function is a positive non-decreasing convex function $\Psi:[0, \infty) \rightarrow$ $[0, \infty)$ such that $\Psi(0)=0$ and $\Psi(\infty)=\infty$. If $\mu$ is a positive measure on $\mathbb{D}$, the Orlicz space $L^{\Psi}(\mu)$ is the space of (classes of) measurable functions $f: \mathbb{D} \rightarrow \mathbb{C}$ such that $\int_{\mathbb{D}} \Psi(|f| / C) d \mathcal{A}<\infty$ for some constant $C>0$, and the norm $\|f\|_{\Psi}$ is defined as the infimum of all constants $C>0$ for which $\int_{\mathbb{D}} \Psi(|f| / C) d \mathcal{A} \leq 1$. The Bergman-Orlicz space is the subspace of $L^{\Psi}(\mathcal{A})$ whose members are analytic in $\mathbb{D}$.

The Hardy-Orlicz space $H^{\Psi}$ is the subspace of $H^{1}$ whose boundary values are in the Orlicz space $L^{\Psi}(\mathbb{T}, m)$.

We refer to [3] (see also [5], and [20]) for the theory of Bergman spaces and to 17] for more information about Orlicz spaces.

\section{Carleson embeddings}

In this section we consider the "embedding" map $I_{\mu}: \mathfrak{B}^{\Psi_{1}} \rightarrow L^{\Psi_{2}}(\mu)$, defined by $I_{\mu}(f)=f$, where $\mu$ is an arbitrary finite positive Borel measure on $\mathbb{D}$ and $\Psi_{1}$ and $\Psi_{2}$ are two Orlicz functions.

\subsection{Boundedness.}

Theorem 2.1. Given $\mu$ a finite positive Borel measure on $\mathbb{D}$ and $\Psi_{1}$ and $\Psi_{2}$ two Orlicz functions, let $I_{\mu}$ be the canonical map defined by $I_{\mu}(f)=f$. One has:

1) If $I_{\mu}: \mathfrak{B}^{\Psi_{1}} \rightarrow L^{\Psi_{2}}(\mu)$ is defined and bounded, then there is a constant $A>0$ such that

$$
\rho_{\mu}(h) \leq \frac{1}{\Psi_{2}\left[A \Psi_{1}^{-1}\left(1 / h^{2}\right)\right]}, \quad \text { for all } 0<h<1
$$


2) In order that $I_{\mu}: \mathfrak{B}^{\Psi_{1}} \rightarrow L^{\Psi_{2}}(\mu)$ be defined and bounded, it suffices that there is a constant $A>0$ such that

$$
K_{\mu, 2}(h) \leq \frac{1 / h^{2}}{\Psi_{2}\left[A \Psi_{1}^{-1}\left(1 / h^{2}\right)\right]}, \quad \text { for all } 0<h<1 .
$$

Note that condition (2.1) reads as $\frac{\Psi_{1}^{-1}\left(1 / h^{2}\right)}{\Psi_{2}^{-1}\left(1 / \rho_{\mu}(h)\right)}$ is bounded (by $1 / A$ ) and condition (2.2) as $\frac{\Psi_{1}^{-1}\left(1 / h^{2}\right)}{\Psi_{2}^{-1}\left(1 / h^{2} K_{\mu, 2}(h)\right)}$ is bounded.

When $\Psi_{1}=\Psi_{2}=\Psi$ and the Orlicz function $\Psi$ satisfies the usual condition $\Delta_{2}: \Psi(2 x) \leq C \Psi(x)$ for some constant $C>1$ and $x$ large enough, it is clear that conditions (2.1) and (2.2) are equivalent. However, they are not equivalent in general, and even condition (2.1) is not sufficient and condition (2.2) is not necessary: the examples $\mathbf{1 . b}$ and 2. of [7], Chapter 4, $\S 3$, given in the Hardy case, also work for the Bergman case. For the sake of completeness, we are going to sketch them 1

Example 1. For every Orlicz function which does not satisfy the $\Delta_{2}$ condition, there exists a finite positive measure $\mu$ on $\mathbb{D}$ such that $I_{\mu}: \mathfrak{B}^{\Psi} \rightarrow L^{\Psi}(\mu)$ is continuous, although $\mu$ is not a 2 -Carleson measure, and a fortiori does not verify (2.2).

Proof. Since $\Psi$ does not satisfy $\Delta_{2}$, there exists an increasing sequence $\left(a_{n}\right)_{n \geq 1}$ such that $\Psi\left(2 a_{n}\right) / n$ is increasing and $\Psi\left(2 a_{n}\right) / \Psi\left(a_{n}\right) \geq n 2^{n}$. Define the discrete measure $\mu$,

$$
\mu=\sum_{n=1}^{\infty}\left(\frac{n}{\Psi\left(2 a_{n}\right)}-\frac{n+1}{\Psi\left(2 a_{n+1}\right)}\right) \delta_{x_{n}},
$$

where $x_{n}=1-1 / \sqrt{\Psi\left(2 a_{n}\right)}$. As $\mu\left(\left[x_{N}, 1\right]\right)=N / \Psi\left(2 a_{N}\right)$, $\mu$ is not a 2-Carleson measure. On the other hand, for every $f$ in the unit ball of $\mathfrak{B}^{\Psi}$, one has (7], Lemma 5.2) $|f(z)| \leq 8 \Psi^{-1}\left[1 /(1-|z|)^{2}\right]$, and it is easy to check that if $g(z)=$ $\Psi^{-1}\left[1 /(1-|z|)^{2}\right]$, then $\|g\|_{L^{\Psi}(\mu)} \leq 2$, so $\|f\|_{L^{\Psi}(\mu)} \leq 16$, proving that $I_{\mu}$ is bounded.

Example 2. Let $\Psi(x)=\mathrm{e}^{x}-1$. There exists a finite positive measure $\mu$ on $\mathbb{D}$ such that (2.1) holds but $I_{\mu}: \mathfrak{B}^{\Psi} \rightarrow L^{\Psi}(\mu)$ is not bounded.

Proof. Let $\nu$ be a probability measure on $\mathbb{T}$, supported by a compact set $L$ of Lebesgue measure zero, such that $\nu(I) \leq|I|^{1 / 2}$, for each interval $I$. We can associate to $\nu$ the measure on $\overline{\mathbb{D}}$ defined by $\tilde{\nu}(E)=\nu(E \cap \mathbb{T})$. By Rudin-Carleson's Theorem, for every integer $n$, there exists a function $g_{n}$ in the unit ball of the disk algebra such that $\left|g_{n}\right|=1$ on $L$ and $\left\|g_{n}\right\|_{H^{\Psi}} \leq 4^{-n}$. As $L$ is compact, there exists some $r_{n} \in(1 / 2,1)$ such that $\left|g_{n}\left(r_{n} z\right)\right| \geq 1 / 2$ for every $z \in L$. Now, define the measure $\mu$ by

$$
\mu(E)=\sum_{n=1}^{\infty} \frac{1}{2^{n}} \nu_{n}(E),
$$

\footnotetext{
${ }^{1}$ Since this paper was submitted, the following paper was published: Carleson measure in Bergman-Orlicz space of polydisc, Abstr. Appl. Anal. 2010, Art. ID 603968, by A.-J. Xu and $\mathrm{Z}$. Yang. It is asserted that the existence and boundedness of the inclusion map $I_{\mu}$ (for $\left.\Psi_{1}=\Psi_{2}=\Psi\right)$ is equivalent to 2.1, but there is a gap in the proof.
} 
where

$$
\nu_{n}(E)=\nu\left(\left\{z \in \mathbb{T} ; r_{n} z \in E\right\}\right) .
$$

If $W$ is a Carleson window of size $h$, then for each $n \geq 1$, we have

$$
\nu\left(\left\{z \in \mathbb{T} ; r_{n} z \in W\right\}\right) \leq \nu(W \cap \mathbb{T}) \leq(2 h)^{1 / 2} .
$$

Hence, $\mu(W) \leq(2 h)^{1 / 2} \lesssim 1 / \Psi\left[\frac{1}{4} \Psi^{-1}\left(1 / h^{2}\right)\right]$, and condition (2.1) is fulfilled.

Nevertheless, the identity from $\mathfrak{B}^{\Psi}$ to $L^{1}(\mu)$ is not continuous, since this would imply that the identity from $H^{\Psi}$ to $L^{1}(\mu)$ is continuous as well, which is not the case: $\left\|g_{n}\right\|_{L^{1}(\mu)} \geq 1 / 2^{n+1}$.

In order to prove Theorem 2.1, we shall need some results. They are analogous to Proposition 4.9, Theorem 4.13 and Lemma 4.14 of [7], but their proofs require different arguments 2

We first introduce the following maximal function:

$$
\Lambda_{f}=\sum_{k=0}^{\infty}\left(\sup _{\Delta_{k}}|f|\right) \mathbb{1}_{\Delta_{k}}
$$

One has:

Lemma 2.2. For every Orlicz function $\Psi$, the map $f \in \mathfrak{B}^{\Psi} \mapsto \Lambda_{f} \in L^{\Psi}(\mathbb{D})$ is bounded.

Proof. Fix $f \in \mathfrak{B}^{\Psi}$. Set $c_{k}=\sup _{\Delta_{k}}|f|$ for every $k \geq 1$, and let $\alpha_{k} \in \Delta_{k}$ be such that $\left|f\left(\alpha_{k}\right)\right| \geq \frac{1}{2} \sup _{\Delta_{k}}|f|=c_{k} / 2$. With $C=\|f\|_{\mathfrak{B}^{\Psi}}>0$, one has

$$
\int_{\mathbb{D}} \Psi\left(\Lambda_{f} / 2 C\right) d \mathcal{A}=\sum_{k \geq 0} \Psi\left(c_{k} / 2 C\right) \mathcal{A}\left(\Delta_{k}\right) \leq \int_{\mathbb{D}} \Psi(|f| / C) d \mu,
$$

where $\mu=\sum_{k \geq 0} \mathcal{A}\left(\Delta_{k}\right) \delta_{\alpha_{k}}$.

But, for every Carleson window $W$, we can write

$$
\mu(W)=\sum_{\alpha_{k} \in W} \mathcal{A}\left(\Delta_{k}\right) \leq \sum_{\Delta_{k} \cap W \neq \emptyset} \mathcal{A}\left(\Delta_{k}\right)=\mathcal{A}\left(\bigcup_{\Delta_{k} \cap W \neq \emptyset} \Delta_{k}\right),
$$

and, since $\bigcup_{\Delta_{k} \cap W \neq \emptyset} \Delta_{k}$ is contained in the window $\tilde{W}$ with the same center as $W$, but with size two times that of $W$, one has $\mu(W) \leq \mathcal{A}(\tilde{W})=4 \mathcal{A}(W)$. Hence $\mu$ is a Bergman-Carleson measure. By [4], it follows that, for some constant $C_{0}>0$, which does not depend on $f$, one has, using the subharmonicity of $\Psi(|f| / C)$,

$$
\int_{\mathbb{D}} \Psi(|f| / C) d \mu \leq C_{0} \int_{\mathbb{D}} \Psi(|f| / C) d \mathcal{A} \leq C_{0} .
$$

We shall, as we may, assume that $C_{0} \geq 1$. Now, by convexity of $\Psi$, we get

$$
\int_{\mathbb{D}} \Psi\left(\frac{\Lambda_{f}}{2 C_{0}\|f\|_{\mathfrak{B}^{\Psi}}}\right) d \mathcal{A} \leq \int_{\mathbb{D}} \frac{1}{C_{0}} \Psi\left(\frac{\Lambda_{f}}{2\|f\|_{\mathfrak{B}^{\Psi}}}\right) d \mathcal{A} \leq 1
$$

meaning that $\left\|\Lambda_{f}\right\|_{L^{\Psi}(\mathbb{D})} \leq 2 C_{0}\|f\|_{\mathfrak{B}^{\Psi}}$.

\footnotetext{
${ }^{2}$ By the way, we seize the opportunity here to correct the proof of Theorem 4.13 given in [7, where some argument had been awkwardly put. In that proof, we first had to set $\mathcal{M}=\{z \in$ $\overline{\mathbb{D}} ;|z|>1-h$ and $|f(z)|>t\}$. Then, $M_{f}$ being the non-tangential maximal function of $f \in H^{1}$, the open set $\left\{M_{f}>t\right\}$ is the disjoint union of a countable family of open $\operatorname{arcs} I_{j} \subseteq \mathbb{T}$, and we had to say that every $z$ such that $|f(z)|>t$ belongs to some window $W\left(I_{j}\right)$ (see [2], page 39).
} 
Lemma 2.3. For every $f \in \mathfrak{B}^{1}$ and every finite positive Borel measure $\mu$ on $\mathbb{D}$, one has, for $0<h<1 / 2$ and $t>0$,

$$
\mu(\{z \in \mathbb{D} ;|z|>1-h \quad \text { and } \quad|f(z)|>t\}) \leq 4 K_{\mu, 2}(2 h) \mathcal{A}\left(\left\{\Lambda_{f}>t\right\}\right) .
$$

Proof. Observe that when $z \in \Delta_{k}$ and $|z|>1-h$, we must have $1-2^{-n-1}>|z|>$ $1-h$, hence $h>2^{-n-1}$. Since $k=2^{n}+j-1 \geq 2^{n-1}$, we must have $k \geq 1 / 4 h$. Let $I=\left\{k \geq 1 ; \sup _{\Delta_{k}}|f|>t\right\}$ and $I_{h}=\left\{k \geq 1 / 4 h ; \sup _{\Delta_{k}}|f|>t\right\}$. If $W_{k}$ is the smallest Carleson window containing $\Delta_{k}$, we have

$$
\begin{aligned}
\mu(\{z \in \mathbb{D} ;|z|>1-h & \text { and }|f(z)|>t\}) \\
& \leq \sum_{k \in I_{h}} \mu\left(\Delta_{k}\right) \leq \sum_{k \in I_{h}, k \geq 1 / 4 h} \mu\left(W_{k}\right) \\
& \lesssim \sum_{k \in I_{h}} K_{\mu, 2}(2 h) \mathcal{A}\left(W_{k}\right) \leq 4 \sum_{k \in I_{h}} K_{\mu, 2}(2 h) \mathcal{A}\left(\Delta_{k}\right) \\
& \leq 4 K_{\mu, 2}(2 h) \sum_{k \in I} \mathcal{A}\left(\Delta_{k}\right)=4 K_{\mu, 2}(2 h) \mathcal{A}\left(\left\{\Lambda_{f}>t\right\}\right),
\end{aligned}
$$

and Lemma 2.3 is proved.

Lemma 2.4. Let $\mu$ be a finite Borel measure on $\mathbb{D}$ and $\Psi_{1}$ and $\Psi_{2}$ be two Orlicz functions.

We suppose that, for some positive constant $A$, there is $0<h_{A} \leq 1 / 2$ such that

$$
K_{\mu, 2}(h) \leq \frac{1 / h^{2}}{\Psi_{2}\left[A \Psi_{1}^{-1}\left(1 / h^{2}\right)\right]}, \quad \text { for } \quad 0<h<h_{A} .
$$

Then, for every $f \in \mathfrak{B}^{\Psi_{1}}$ such that $\|f\|_{\mathfrak{B}^{\Psi_{1}}} \leq 1$ and every Borel subset $E$ of $\mathbb{D}$, one has, with $x_{A}=(A / 8) \Psi_{1}^{-1}\left(4 / h_{A}^{2}\right)$,

$$
\int_{E} \Psi_{2}(A|f| / 64) d \mu \leq \mu(E) \Psi_{2}\left(x_{A}\right)+\frac{1}{8} \int_{\mathbb{D}} \Psi_{1}\left(\Lambda_{f}\right) d \mathcal{A} .
$$

Proof. For every $s>0$, the inequality $|f(z)|>s$ implies that the norm of the evaluation $\delta_{z}$ at $z$ is greater than $s$. However this norm is ([7, Lemma 5.2) $\Psi_{1}\left(1 /(1-|z|)^{2}\right)$, up to constants. More precisely,

$$
\left\|\delta_{z}\right\| \leq 8 \Psi_{1}^{-1}\left(\frac{1}{(1-|z|)^{2}}\right) .
$$

Hence, we have

so

$$
s<8 \Psi_{1}^{-1}\left(\frac{1}{(1-|z|)^{2}}\right)
$$

$$
|z|>1-\frac{1}{\sqrt{\Psi_{1}(s / 8)}} .
$$

Lemma 2.3 gives, when $\Psi_{1}(s / 8) \geq 2$,

$$
\begin{aligned}
\mu(\{|f(z)|>s\}) & =\mu\left(\left\{|z|>1-\frac{1}{\sqrt{\Psi_{1}(s / 8)}} \text { and }|f(z)|>s\right\}\right) \\
& \leq 4 K_{\mu, 2}\left(\frac{2}{\sqrt{\Psi_{1}(s / 8)}}\right) \mathcal{A}\left(\left\{\Lambda_{f}>s\right\}\right) .
\end{aligned}
$$


But, by our assumption, if $\Psi_{1}(s / 8) \geq 4 / h_{A}^{2}$,

$$
\begin{aligned}
K_{\mu, 2}\left(\frac{2}{\sqrt{\Psi_{1}(s / 8)}}\right) & \leq \frac{\Psi_{1}(s / 8) / 4}{\Psi_{2}\left[A \Psi_{1}^{-1}\left(\Psi_{1}(s / 8) / 4\right)\right]} \\
& \leq \frac{\Psi_{1}(s / 8) / 4}{\Psi_{2}\left[(A / 4) \Psi_{1}^{-1}\left(\Psi_{1}(s / 8)\right)\right]} \quad \text { (by convexity) } \\
& =\frac{1}{4} \frac{\Psi_{1}(s / 8)}{\Psi_{2}(A s / 32)} ;
\end{aligned}
$$

hence

$$
\mu(\{|f(z)|>s\}) \leq \frac{\Psi_{1}(s / 8)}{\Psi_{2}(A s / 32)} \mathcal{A}\left(\left\{\Lambda_{f}>s\right\}\right) .
$$

We therefore obtain

$$
\begin{aligned}
\int_{E} \Psi_{2}(A|f| / 64) d \mu & =\int_{0}^{+\infty} \Psi_{2}^{\prime}(t) \mu(\{|f|>64 t / A\} \cap E) d t \\
\leq & \int_{0}^{x_{A}} \Psi_{2}^{\prime}(t) \mu(E) d t \\
& \quad+\int_{x_{A}}^{+\infty} \Psi_{2}^{\prime}(t) \frac{\Psi_{1}(8 t / A)}{\Psi_{2}(2 t)} \mathcal{A}\left(\left\{\Lambda_{f}>64 t / A\right\}\right) d t \\
\leq & \Psi_{2}\left(x_{A}\right) \mu(E) \\
& \quad+\int_{x_{A}}^{+\infty} \frac{\Psi_{2}^{\prime}(t)}{\Psi_{2}(2 t)} \Psi_{1}(8 t / A) \mathcal{A}\left(\left\{\Lambda_{f}>64 t / A\right\}\right) d t .
\end{aligned}
$$

But, as $\Psi_{1}$ and $\Psi_{2}$ are Orlicz functions, one has $t \Psi_{2}^{\prime}(t) \leq \Psi_{2}(2 t)$ and $\Psi_{1}(8 t / A) \leq$ $(8 t / A) \Psi_{1}^{\prime}(8 t / A)$. Hence

$$
\begin{aligned}
\int_{x_{A}}^{+\infty} \frac{\Psi_{2}^{\prime}(t)}{\Psi_{2}(2 t)} \Psi_{1}(8 t / A) & \mathcal{A}\left(\left\{\Lambda_{f}>64 t / A\right\}\right) d t \\
& \leq \int_{0}^{+\infty} \frac{\Psi_{1}(8 t / A)}{t} \mathcal{A}\left(\left\{\Lambda_{f}>64 t / A\right\}\right) d t \\
& \leq \frac{8}{A} \int_{0}^{+\infty} \Psi_{1}^{\prime}(8 t / A) \mathcal{A}\left(\left\{\Lambda_{f}>64 t / A\right\}\right) d t \\
& =\int_{0}^{+\infty} \Psi_{1}^{\prime}(x) \mathcal{A}\left(\left\{\Lambda_{f}>8 x\right\}\right) d x \\
& =\int_{\mathbb{D}} \Psi_{1}\left(\Lambda_{f} / 8\right) d \mathcal{A} \leq \frac{1}{8} \int_{\mathbb{D}} \Psi_{1}\left(\Lambda_{f}\right) d \mathcal{A}
\end{aligned}
$$

and the proof of Lemma 2.4 is finished.

Proof of Theorem 2.1. 1) Consider, for every $a \in \mathbb{D}$, the Berezin kernel

$$
H_{a}(z)=\frac{\left(1-|a|^{2}\right)^{2}}{|1-\bar{a} z|^{4}} .
$$

One has $\left\|H_{a}\right\|_{\mathfrak{B}^{1}}=1$ and

$$
\left\|H_{a}\right\|_{\infty}=\frac{\left(1-|a|^{2}\right)^{2}}{(1-|a|)^{4}}=\frac{(1+|a|)^{2}}{(1-|a|)^{2}} \leq \frac{4}{(1-|a|)^{2}} ;
$$


hence ([7, Lemma 3.9)

$$
\left\|H_{a}\right\|_{\mathfrak{B}^{\Psi_{1}}} \leq \frac{4 / h^{2}}{\Psi_{1}^{-1}\left(4 / h^{2}\right)}, \quad h=1-|a| .
$$

It follows that the function $f_{a}=\frac{1}{4} h^{2} \Psi_{1}^{-1}\left(4 / h^{2}\right) H_{a}$ is in the unit ball of $\mathfrak{B}^{\Psi_{1}}$.

Now, let $\xi \in \mathbb{T}$ and $0<h<1$. When $z \in W(\xi, h)$, one easily has (see [7, proof of Theorem 4.10, with a slightly different definition of $W(\xi, h))|1-\bar{a} z| \leq 5 h$, where $a=(1-h) \xi$. It then follows that $\left|f_{a}(z)\right| \geq(1 / 2500) \Psi_{1}^{-1}\left(4 / h^{2}\right)$. Hence

$$
1 \geq \int_{\mathbb{D}} \Psi_{2}\left(\frac{\left|f_{a}\right|}{\left\|I_{\mu}\right\|}\right) d \mu \geq \Psi_{2}\left(\frac{1}{2500\left\|I_{\mu}\right\|} \Psi_{1}^{-1}\left(4 / h^{2}\right)\right) \mu(W(\xi, h)),
$$

which is 2.1.

2) By Lemma 2.2, there is a constant $C>0$, that we may, and shall, assume $\geq 1$, such that $\left\|\Lambda_{f}\right\|_{L^{\Psi_{1}(\mu)}} \leq C\|f\|_{\mathfrak{B}^{\Psi_{1}}}$ for every $f \in \mathfrak{B}^{\Psi_{1}}$. Let $g$ be in the unit ball of $\mathfrak{B}^{\Psi_{1}}$, and apply Lemma 2.4 to $f=g / C$ (whose norm is $\leq 1$ yet), $E=\mathbb{D}$, with $h_{A}=1 / 2$. We get, with $\tilde{C}=\max \left(1, \mu(\mathbb{D}) \Psi_{2}\left(x_{A}\right)+\frac{1}{8}\right)$,

$$
\begin{aligned}
\int_{\mathbb{D}} \Psi_{2}\left(\frac{A}{64 C \tilde{C}}|g|\right) d \mu & \leq \frac{1}{\tilde{C}} \int_{\mathbb{D}} \Psi_{2}\left(\frac{A}{64 C}|g|\right) d \mu \\
& \leq \frac{1}{\tilde{C}}\left[\mu(\mathbb{D}) \Psi_{2}\left(x_{A}\right)+\frac{1}{8} \int_{\mathbb{D}} \Psi_{1}\left(\Lambda_{f} / C\right) d \mathcal{A}\right] \\
& \leq \frac{1}{\tilde{C}}\left[\mu(\mathbb{D}) \Psi_{2}\left(x_{A}\right)+\frac{1}{8}\right] \leq 1,
\end{aligned}
$$

which means that $\|g\|_{L^{\Psi_{2}(\mu)}} \leq 64 C \tilde{C} / A$.

\subsection{Compactness.}

Theorem 2.5. Let $\mu$ be a finite positive Borel measure on $\mathbb{D}, \Psi_{1}$ and $\Psi_{2}$ be two Orlicz functions, and let $I_{\mu}: \mathfrak{B}^{\Psi_{1}} \rightarrow L^{\Psi_{2}}(\mu)$ be the canonical map defined by $I_{\mu}(f)=f$. One has:

1) If $I_{\mu}$ is compact, then

$$
\lim _{h \rightarrow 0} \frac{\Psi_{1}^{-1}\left(1 / h^{2}\right)}{\Psi_{2}^{-1}\left(1 / \rho_{\mu}(h)\right)}=0 .
$$

2) In order that $I_{\mu}$ be compact, it suffices that

$$
\lim _{h \rightarrow 0} \frac{\Psi_{1}^{-1}\left(1 / h^{2}\right)}{\Psi_{2}^{-1}\left(1 / h^{2} K_{\mu, 2}(h)\right)}=0 .
$$

As for the boundedness case, conditions (2.6) and (2.7) are equivalent if $\Psi_{1}=$ $\Psi_{2}=\Psi$ is sufficiently regular, but not in general. We shall give examples after the proof of the theorem, at the end of the section.

To prove the first part of this theorem, we shall need the following lemma.

Lemma 2.6. $I_{\mu}: \mathfrak{B}^{\Psi_{1}} \rightarrow L^{\Psi_{2}}(\mu)$ is compact if and only if for every bounded sequence $\left(f_{n}\right)_{n}$ in $\mathfrak{B}^{\Psi_{1}}$ converging to 0 uniformly on compact subsets of $\mathbb{D}$, the sequence $\left(I_{\mu}\left(f_{n}\right)\right)_{n}$ converges to 0 in the norm of $L^{\Psi_{2}}(\mu)$.

Proof of Lemma 2.6. Assume that $I_{\mu}$ is compact, and let $\left(f_{n}\right)_{n}$ be a bounded sequence in $\mathfrak{B}^{\Psi_{1}}$ which converges to 0 uniformly on compact subsets of $\mathbb{D}$. Since $I_{\mu}$ is compact and $\left(f_{n}\right)_{n}$ is bounded, we have a subsequence $\left(g_{n}\right)_{n}$ such that $g_{n}=I_{\mu}\left(g_{n}\right)$ 
converges to some $g \in L^{\Psi_{2}}(\mu)$. Then some subsequence of $\left(g_{n}\right)_{n}$ converges $\mu$-a.e. to $g$. Since $\left(g_{n}\right)_{n}$ converges to 0 uniformly on compact subsets of $\mathbb{D}$, we must have $g=0 \mu$-a.e., that is, $g=0$ as an element of $L^{\Psi_{2}}(\mu)$. Now, the compactness of $I_{\mu}$ implies that $\left\|f_{n}\right\|_{L^{\Psi_{2}(\mu)}}$ tends to 0 .

Conversely, assume that the condition of the lemma is satisfied, and let $\left(f_{n}\right)_{n}$ be an arbitrary bounded sequence in $\mathfrak{B}^{\Psi_{1}}$. Since the evaluation map is continuous on $\mathfrak{B}^{\Psi_{1}}$ ([7], Lemma 5.2), $\left(f_{n}\right)_{n}$ is a normal family, and Montel's Theorem gives a subsequence $\left(g_{n}\right)_{n}$ which converges uniformly on compact subsets to some holomorphic function $g$. By Fatou's lemma, $g$ belongs to $\mathfrak{B}^{\Psi_{1}}$. Now, $\left(g_{n}-g\right)_{n}$ is a bounded sequence of $\mathfrak{B}^{\Psi_{1}}$ which converges to 0 uniformly on compact subsets of $\mathbb{D}$. By hypothesis, $\left\|g_{n}-g\right\|_{L^{\Psi_{2}(\mu)}}$ tends to 0 , and it follows that $I_{\mu}$ is compact.

Proof of Theorem 2.5, 1) Assume that the map $I_{\mu}: \mathfrak{B}^{\Psi_{1}} \rightarrow L^{\Psi_{2}}(\mu)$ is compact. Consider, for every $a \in \mathbb{D}$, the Berezin kernel (2.4). It follows from (2.4) and (2.5) that $H_{a} /\left\|H_{a}\right\|_{\mathfrak{B}^{\Psi_{1}}}$ converges uniformly to 0 on compact subsets of $\mathbb{D}$ as $|a|$ goes to 1 . Hence, by Lemma 2.6. the compactness of $I_{\mu}$ implies that $\left\|\left(H_{a} /\left\|H_{a}\right\|_{\mathfrak{B}^{\Psi_{1}}}\right)\right\|_{L^{\Psi_{2}}(\mu)}$ tends to 0 . That means that, for every $\varepsilon>0$, one has

$$
\int_{\mathbb{D}} \Psi_{2}\left(\frac{\left|H_{a}\right|}{\varepsilon\left\|H_{a}\right\|_{\mathfrak{B}^{\Psi_{1}}}}\right) d \mu \leq 1
$$

for $|a|$ close enough to 1 , depending on $\varepsilon$.

Now, let $\xi \in \mathbb{T}$ and $0<h<1$ with $a=(1-h) \xi$. As already said in the proof of Theorem 2.1 $z \in W(\xi, h)$ implies that $\left|H_{a}(z)\right| \geq 1 / 625 h^{2}$. Therefore,

$$
\int_{\mathbb{D}} \Psi_{2}\left(\frac{\left|H_{a}\right|}{\varepsilon\left\|H_{a}\right\|_{\mathfrak{B}^{\Psi_{1}}}}\right) d \mu \geq \Psi_{2}\left(\frac{1 / 625 h^{2}}{\varepsilon\left(4 / h^{2}\right) / \Psi_{1}^{-1}\left(4 / h^{2}\right)}\right) \mu(W(\xi, h)) .
$$

We get, for $h>0$ small enough,

$$
\mu(W(\xi, h)) \leq \frac{1}{\Psi_{2}\left((1 / 2500 \varepsilon) \Psi_{1}^{-1}\left(4 / h^{2}\right)\right)} .
$$

Since $\xi \in \mathbb{T}$ is arbitrary, it follows that, for $h>0$ small enough,

$$
\rho_{\mu}(h) \leq \frac{1}{\Psi_{2}\left((1 / 2500 \varepsilon) \Psi_{1}^{-1}\left(4 / h^{2}\right)\right)},
$$

which reads

$$
\frac{\Psi_{1}^{-1}\left(4 / h^{2}\right)}{\Psi_{2}^{-1}\left(1 / \rho_{\mu}(h)\right)} \leq 2500 \varepsilon .
$$

Since $\Psi_{1}^{-1}\left(4 / h^{2}\right) \geq \Psi_{1}^{-1}\left(1 / h^{2}\right)$, we have obtained (2.6).

2) Assume now that (2.7) is satisfied. By Lemma 2.6, we have to show that for every sequence $\left(f_{n}\right)_{n}$ in the unit ball of $\mathfrak{B}^{\Psi_{1}}$ which converges uniformly to 0 on compact subsets of $\mathbb{D},\left(I_{\mu}\left(f_{n}\right)\right)_{n}$ converges to 0 for the norm of $L^{\Psi_{2}}(\mu)$. So, let $\left(f_{n}\right)_{n}$ be such a sequence, and let $\varepsilon>0$.

By Lemma 2.2. there is a constant $C \geq 1$ such that $\left\|\Lambda_{f}\right\|_{L^{\Psi_{1}(\mu)}} \leq C\|f\|_{\mathfrak{B}^{\Psi_{1}}}$ for every $f \in \mathfrak{B}^{\Psi_{1}}$. Set $A=64 C / \varepsilon$. By (2.7), there is an $h_{A}<1$ such that, for $0<h \leq h_{A}$, one has $\Psi^{-1}\left(1 / h^{2}\right) \leq(1 / A) \Psi_{2}^{-1}\left(1 / h^{2} K_{\mu, 2}(h)\right)$, i.e.

$$
K_{\mu, 2}(h) \leq \frac{1 / h^{2}}{\Psi_{2}\left[A \Psi_{1}^{-1}\left(1 / h^{2}\right)\right]} .
$$


For $0<r<1$, we may therefore apply, for every $n \geq 1$, Lemma 2.4 to $f_{n} / C$ (which is in the unit ball of $\mathfrak{B}^{\Psi_{1}}$ ), with $E=\mathbb{D} \backslash r \overline{\mathbb{D}}$. We get

$$
\begin{aligned}
\int_{\mathbb{D} \backslash r \overline{\mathbb{D}}} \Psi_{2}\left(\left|f_{n}\right| / \varepsilon\right) d \mu & =\int_{\mathbb{D} \backslash r \overline{\mathbb{D}}} \Psi_{2}\left(A\left|f_{n}\right| / 64 C\right) d \mu \\
& \leq \mu(\mathbb{D} \backslash r \overline{\mathbb{D}}) \Psi_{2}\left(x_{A}\right)+\frac{1}{8} \int_{\mathbb{D}} \Psi_{1}\left(\Lambda_{f_{n}} / C\right) d \mathcal{A} \\
& \leq \mu(\mathbb{D} \backslash r \overline{\mathbb{D}}) \Psi_{2}\left(x_{A}\right)+\frac{1}{8} .
\end{aligned}
$$

But this last quantity is $\leq 1 / 2$ for $r$ small enough.

Fix such an $r<1$. Since $\left(f_{n}\right)_{n}$ converges uniformly to 0 on compact subsets of $\mathbb{D}$, one has $\int_{r \overline{\mathbb{D}}} \Psi_{2}\left(\left|f_{n}\right| / \varepsilon\right) d \mu \leq 1 / 2$ for $n$ large enough.

It follows that $\int_{\mathbb{D}} \Psi_{2}\left(\left|f_{n}\right| / \varepsilon\right) d \mu \leq 1$, and hence $\left\|f_{n}\right\|_{L^{\Psi_{2}}}(\mu) \leq \varepsilon$, for $n$ large enough.

That completes the proof of Theorem 2.5 .

As we said, in general, condition (2.6) is not sufficient to ensure compactness, and condition (2.7) is not necessary. They are equivalent (and hence necessary and sufficient for compactness) if $\Psi_{1}=\Psi_{2}=\Psi$ and $\Psi$ is a regular Orlicz function. Here, regular means that $\Psi$ satisfies the condition we called $\nabla_{0}$ : for some $x_{0}>0$ and some $C \geq 1$, one has $\frac{\Psi(2 x)}{\Psi(x)} \leq \frac{\Psi(2 C y)}{\Psi(y)}$ for $x_{0} \leq x \leq y$ (see [7, Theorem 4.11, whose proof works as well in the Bergman case). However, in 7] we gave some examples showing, in the Hardy case, that this is not always the case (examples $\mathbf{3}$ and 4 in [7], Chapter $4, \S 3)$. These examples work in the Bergman case, and we are going to recall them sketchily.

Example 1. For every Orlicz function $\Psi$ not satisfying $\nabla_{0}$, there exists a measure $\mu$ such that $I_{\mu}: \mathfrak{B}^{\Psi} \rightarrow L^{\Psi}(\mu)$ is compact but for which (2.7) is not satisfied.

Proof. Since $\Psi \notin \nabla_{0}$, we can select two increasing sequences $\left(x_{n}\right)_{n \geq 1}$ and $\left(y_{n}\right)_{n \geq 1}$, with $1 \leq x_{n} \leq y_{n} \leq x_{n+1}$ and $\Psi\left(x_{n}\right)>1$, such that $\lim x_{n}=+\infty$ and

$$
\frac{\Psi\left(2 x_{n}\right)}{\Psi\left(x_{n}\right)} \geq \frac{\Psi\left(2^{n} y_{n}\right)}{\Psi\left(y_{n}\right)} .
$$

Define the discrete measure

$$
\mu=\sum_{n=1}^{\infty} \frac{1}{\Psi\left(2^{n} y_{n}\right)} \delta_{r_{n}}
$$

where $r_{n}=1-1 / \sqrt{\Psi\left(y_{n}\right)}$. The series converge since $\Psi\left(2^{n} y_{n}\right) \geq 2^{n}$.

The same proof as in [7] shows that $I_{\mu}$ is compact, but, writing $h_{n}=1 / \sqrt{\Psi\left(x_{n}\right)}$ and $t_{n}=1 / \sqrt{\Psi\left(y_{n}\right)}$, we have

$$
K_{\mu, 2}\left(h_{n}\right) \geq \frac{\mu\left(\left[1-t_{n}, 1\right]\right)}{t_{n}^{2}} \geq \frac{\Psi\left(y_{n}\right)}{\Psi\left(2^{n} y_{n}\right)} \geq \frac{\Psi\left(x_{n}\right)}{\Psi\left(2 x_{n}\right)}=\frac{1 / h_{n}^{2}}{\Psi\left(2 \Psi^{-1}\left(1 / h_{n}^{2}\right)\right)},
$$

showing that (2.7) is not satisfied.

This actually shows that condition $\Psi \in \nabla_{0}$ is necessary and sufficient in order to ensure that the identity from $\mathfrak{B}^{\Psi}$ to $L^{\Psi}(\mu)$ is compact if and only if $\mu$ satisfies (2.7). 
Example 2. There exist an Orlicz function $\Psi$ and a measure $\mu$ on $\mathbb{D}$ such that (2.6) holds, but for which $I_{\mu}: \mathfrak{B}^{\Psi} \rightarrow L^{\Psi}(\mu)$ is not compact.

Proof. We shall use the Orlicz function $\Psi$ introduced in [6]. The key properties of this function are:

1) $\Psi(x) \geq x^{3} / 3$ for every $x>0$;

2) $\Psi(k !) \leq(k !)^{3}$ for every integer $k \geq 1$;

3) $\Psi(3(k !))>k \cdot(k !)^{3}$ for every integer $k \geq 1$.

Define $x_{k}=k !, y_{k}=(k+1) ! / k^{1 / 3}, r_{k}=1-1 / \sqrt{\Psi\left(y_{k}\right)}$ and $\rho_{k}=1-1 / \sqrt{\Psi\left(x_{k}\right)}$. Of course, $x_{2}<y_{2}<x_{3}<\cdots$. Let $\nu$ be the discrete measure defined by

$$
\nu=\sum_{k=2}^{\infty} \nu_{k},
$$

where

$$
\nu_{k}=\frac{1}{\Psi((k+1) !)} \sum_{a^{k^{2}=1}} \delta_{r_{k} a} .
$$

In order to show that (2.6) is satisfied, it is clearly sufficient to prove that, when $1 / \sqrt{\Psi\left(y_{k}\right)} \leq h<1 / \sqrt{\Psi\left(y_{k-1}\right)}$ (with $k \geq 3$ ), we have

$$
\rho_{\nu}(h) \leq \frac{1}{\Psi\left(\frac{1}{2} k^{1 / 3} \Psi^{-1}\left(1 / h^{2}\right)\right)} .
$$

But, for such $h$, we have $\Psi^{-1}\left(1 / h^{2}\right) \leq y_{k}$, so

$$
\Psi\left(\frac{1}{2} k^{1 / 3} \Psi^{-1}\left(1 / h^{2}\right)\right) \leq \frac{1}{2} \Psi((k+1) !) .
$$

Hence, the conclusion follows from the fact that $\rho_{\nu}(h) \leq 2 / \Psi((k+1)$ !) (see [7] for more details). So, condition (2.6) is fulfilled.

We now introduce $f_{k}(z)=x_{k}\left(\frac{1-\rho_{k}}{1-\rho_{k} z^{k^{2}}}\right)^{4}$. By (2.5), $\left\|f_{k}\right\|_{\mathfrak{B}^{\Psi}} \leq 1$. An easy computation gives $r_{k}^{k^{2}} \geq \rho_{k}$, for every $k \geq 2$, so, for every $a \in \mathbb{T}$ with $a^{k^{2}}=1$, we have

$$
f_{k}\left(a r_{k}\right) \geq x_{k}\left(\frac{1-\rho_{k}}{1-\rho_{k}^{2}}\right)^{4} \geq \frac{1}{16} x_{k}
$$

Hence

$$
\begin{aligned}
\int_{\mathbb{D} \backslash r_{k-1} \mathbb{D}} \Psi\left(48\left|f_{k}\right|\right) d \nu & \geq \int_{\mathbb{D} \backslash r_{k-1} \mathbb{D}} \Psi\left(48\left|f_{k}\right|\right) d \nu_{k} \geq \frac{k^{2}}{\Psi((k+1) !)} \Psi\left(3 x_{k}\right) \\
& >\frac{k^{2}}{\Psi((k+1) !)}\left(k \cdot(k !)^{3}\right) \geq 1 .
\end{aligned}
$$

Therefore, we conclude that $\sup _{\|f\|_{\mathfrak{B}} \leq 1}\|f\|_{L^{\Psi}\left(\mathbb{D} \backslash r_{k} \mathbb{D}, \mu\right)} \geq 1 / 48$, though $r_{k} \rightarrow 1$. Hence (see the above proof of Theorem 2.5) $I_{\mu}$ is not compact.

\section{COMPACTNESS FOR COMPOSITION OPERATORS}

3.1. Carleson function. We know ([7, Proposition 5.4) that every analytic selfmap $\varphi: \mathbb{D} \rightarrow \mathbb{D}$ induces a bounded composition operator $C_{\varphi}: \mathfrak{B}^{\Psi} \rightarrow \mathfrak{B}^{\Psi}$. The main result of this section is that, for the pull-back measure $\mathcal{A}_{\varphi}$ of $\mathcal{A}$ by $\varphi$, the necessary and the sufficient conditions of Theorem 2.5 are equivalent. The same kind of result occurs for Hardy-Orlicz spaces ([7], Theorem 4.18 and Theorem 4.19), 
but the proofs must be different (because we use the analytic functions themselves and not their boundary values).

We have the following contractivity (or homogeneity) result, which can be viewed as a "multi-scaled" fact that $\mathcal{A}_{\varphi}$ is a 2 -Carleson measure. Since this paper was submitted, we have generalized this theorem for the weighted measures $d \mathcal{A}_{\alpha}(z)=$ $\left(1-|z|^{2}\right)^{\alpha} d \mathcal{A}$, for $\alpha>-1([13])$.

Theorem 3.1. There exists a constant $C_{0}>0$ such that, for every analytic self$\operatorname{map} \varphi: \mathbb{D} \rightarrow \mathbb{D}$, one has

$$
\mathcal{A}(\{\varphi \in S(\xi, \varepsilon h)\}) \leq C_{0} \varepsilon^{2} \mathcal{A}(\{\varphi \in S(\xi, h)\})
$$

for every $\xi \in \mathbb{T}, 0<h<(1-|\varphi(0)|)$, and $0<\varepsilon \leq 1$.

As a consequence, one has $\rho_{\varphi, 2}(\varepsilon h) \leq C \varepsilon^{2} \rho_{\varphi, 2}(h)$, for $h>0$ small enough, and hence

$$
\frac{\rho_{\varphi, 2}(h)}{h^{2}} \leq K_{\mu, 2}(h)=\sup _{0<\varepsilon \leq 1} \frac{\rho_{\varphi, 2}(\varepsilon h)}{\varepsilon^{2} h^{2}} \leq C_{\alpha} \frac{\rho_{\varphi, 2}(h)}{h^{2}}
$$

Therefore

Theorem 3.2. For every analytic self-map $\varphi: \mathbb{D} \rightarrow \mathbb{D}$ and every Orlicz function $\Psi$, the composition operator $C_{\varphi}: \mathfrak{B}^{\Psi} \rightarrow \mathfrak{B}^{\Psi}$ is compact if and only if

$$
\lim _{h \rightarrow 0} \frac{\Psi^{-1}\left(1 / h^{2}\right)}{\Psi^{-1}\left(1 / \rho_{\varphi, 2}(h)\right)}=0 .
$$

Proof of Theorem 3.1. It suffices, even if it means enlarging $C_{0}$, to show (3.1) for $0<h \leq h_{0}=\alpha_{0}(1-|\varphi(0)|)$ and $0<\varepsilon \leq \varepsilon_{0}$ for some $0<\alpha_{0}<1$ and $0<\varepsilon_{0}<1$. Indeed, if $0<h \leq h_{0}$ and $\varepsilon_{0} \leq \varepsilon \leq 1$, one has $\mathcal{A}(S(\xi, \varepsilon h)) \leq \mathcal{A}(S(\xi, h)) \leq$ $\left(1 / \varepsilon_{0}^{2}\right) \varepsilon^{2} \mathcal{A}(S(\xi, h))$. Now, for $h_{0} \leq h<1-|\varphi(0)|$, one has, on the one hand, for $0<\varepsilon \leq \alpha_{0}, \mathcal{A}(S(\xi, \varepsilon h)) \leq \mathcal{A}\left(S\left(\xi,\left(\varepsilon / \alpha_{0}\right) h_{0}\right)\right) \leq C_{0}\left(\varepsilon^{2} / \alpha_{0}^{2}\right) \mathcal{A}\left(S\left(\xi, h_{0}\right)\right) \leq$ $\left(C_{0} / \alpha_{0}^{2}\right) \varepsilon^{2} \mathcal{A}(S(\xi, h))$, and, on the other hand, for $\alpha_{0} \leq \varepsilon \leq 1, \mathcal{A}(S(\xi, \varepsilon h)) \leq$ $\mathcal{A}(S(\xi, h)) \leq\left(1 / \alpha_{0}^{2}\right) \varepsilon^{2} \mathcal{A}(S(\xi, h))$.

Since, moreover, it suffices to make the proof for $\xi=1$, Theorem 3.1 will result from the following theorem.

Theorem 3.3. There exist a constant $K>0, \alpha_{0}>0$ and $\lambda_{0}>1$ such that every analytic function $f: \mathbb{D} \rightarrow \Pi^{+}$with $|f(0)| \leq \alpha_{0}$ satisfies, for every $\lambda \geq \lambda_{0}$,

$$
\mathcal{A}(\{|f|>\lambda\}) \leq \frac{K}{\lambda^{2}} \mathcal{A}(\{|f|>1\}),
$$

where $\Pi^{+}$is the right half-plane $\Pi^{+}=\{z \in \mathbb{C} ; \mathfrak{R e} z>0\}$.

Indeed, let $f=h /(1-\varphi)$. Then $\mathfrak{R e} f>0$ and $|f(0)| \leq h /(1-|\varphi(0)|) \leq \alpha_{0}$. We may apply Theorem 3.3 and we get, for $0<\varepsilon \leq 1 / \lambda_{0}$,

$$
\begin{aligned}
\mathcal{A}_{\varphi}(S(1, \varepsilon h)) & =\mathcal{A}(\{|\varphi-1|<\varepsilon h \mid\})=\mathcal{A}(\{|f|>1 / \varepsilon\}) \\
& \leq K \varepsilon^{2} \mathcal{A}(\{|f|>1\})=K \varepsilon^{2} \mathcal{A}(\{|1-\varphi|<h\})=K \varepsilon^{2} \mathcal{A}_{\varphi}(S(1, h)),
\end{aligned}
$$

which proves Theorem 3.1 


\subsubsection{Some lemmas.}

Lemma 3.4. There is some constant $C_{1}>0$ such that

$$
\mathcal{A}(\{|f|>\lambda\}) \leq \frac{C_{1}}{\lambda^{2}}|f(0)|^{2},
$$

for every analytic function $f: \mathbb{D} \rightarrow \Pi^{+}$and for every $\lambda>0$.

In particular, there is a constant $K_{1}>0$ such that $\|f\|_{L^{1}(\mathbb{D})} \leq K_{1}|f(0)|$ for every such function.

Proof. We may assume that $|f(0)|=1$.

The second assertion follows from the first one:

$$
\int_{\mathbb{D}}|f| d \mathcal{A}=\int_{0}^{+\infty} \mathcal{A}(\{|f|>\lambda\}) d \lambda \leq \int_{0}^{1} d \lambda+\int_{1}^{+\infty} \frac{C_{1}}{\lambda^{2}} d \lambda=1+C_{1}:=K_{1} .
$$

To prove the first assertion, observe first that the left-hand side of inequality (3.3) is $\leq 1$, so (3.3) is obvious for $\lambda \leq 2$ (with $C_{1} \geq 4$ ). Assume that $\lambda>2$, and set $\varphi(z)=\frac{f(z)-f(0)}{f(z)+\overline{f(0)}}$. Then $|f(z)|>\lambda$ implies $|\varphi(z)-1|=2|\mathfrak{R e} f(0)| /|f(z)+\overline{f(0)}| \leq$ $2 /(\lambda-1) \leq 4 / \lambda$. But $\varphi$ maps $\mathbb{D}$ into itself and hence induces a bounded composition operator $C_{\varphi}: \mathfrak{B}^{2} \rightarrow \mathfrak{B}^{2}$. It follows from [4] that $\mathcal{A}_{\varphi}$ is a Bergman-Carleson measure, and hence (see the proof of Theorem 2.1 1), with $\Psi_{1}(x)=\Psi_{2}(x)=x^{2}$ ),

$$
\begin{aligned}
\mathcal{A}(\{|f|>\lambda\}) & \leq \mathcal{A}_{\varphi}(S(1,4 / \lambda)) \leq \mathcal{A}_{\varphi}(W(1,20 / \lambda)) \\
& \leq C_{0}\left\|C_{\varphi}\right\|^{2} /(\lambda / 20)^{2}=C_{0}^{\prime}\left\|C_{\varphi}\right\|^{2} / \lambda^{2},
\end{aligned}
$$

for some constant $C_{0} \leq 2500^{2} / 4$. But $\left\|C_{\varphi}\right\| \leq \frac{1+|\varphi(0)|}{1-|\varphi(0)|}=1$ ([20], Theorem 11.6, page 308$)$, and the result follows.

Let

$$
G=\{z \in \mathbb{C} ;|\arg z|<\pi / 4\} .
$$

By applying Lemma 3.4 to $f^{2}$, we get the following lemma.

Lemma 3.5. There exists a constant $C_{2}>0$ such that for every analytic function $f: \mathbb{D} \rightarrow G$, one has, for every $\lambda>0$,

$$
\mathcal{A}(\{|f|>\lambda\}) \leq \frac{C_{2}}{\lambda^{4}}|f(0)|^{4} .
$$

In particular, there is a constant $K_{2}>0$ such that $\|f\|_{L^{2}(\mathbb{D})} \leq K_{2}|f(0)|$ for every such function.

Now, we shall have to replace $\mathbb{D}$ by some conformal copies of $\mathbb{D}$. This will be possible by the following version of Lemma 3.5

Lemma 3.6. Let $\Omega$ be a bounded Jordan domain bounded by a $\mathcal{C}^{1}$ Jordan curve $J$ and let $h: \mathbb{D} \rightarrow \Omega$ be a Riemann map which extends to a bi-Lipschitz homeomorphism $h$ of $\overline{\mathbb{D}}$ to $\bar{\Omega}$ such that

$$
a \leq\left|h^{\prime}(z)\right| \leq b, \quad \forall z \in \mathbb{D} .
$$

Then, there exists a constant $C>0$, depending only on $b / a$, such that, for any $\lambda>0$ and any analytic function $f: \Omega \rightarrow G$, one has

$$
\mathcal{A}(\{|f|>\lambda\}) \leq \frac{C}{\lambda^{4}} \mathcal{A}(\Omega)|f(c)|^{4}
$$

where $c=h(0)$. 
In particular, there is a constant $K=K(\Omega, c)>0$ such that $\|f\|_{2} \leq K \mathcal{A}(\Omega)|f(c)|$ for all such functions.

Moreover, $C>0$ can be taken so that, for every positive harmonic function $u: \Omega \rightarrow \mathbb{R}_{+}$, one has

$$
\frac{1}{C} u(c) \leq \frac{1}{\mathcal{A}(\Omega)} \int_{\Omega} u d \mathcal{A} \leq C u(c) .
$$

Proof. By the change-of-variable formula, we have, setting $F=f \circ h$ and $w=h(z)$,

$$
\begin{aligned}
\mathcal{A}(\{|f|>\lambda\}) & =\int_{\Omega} \mathbb{1}_{\{|f|>\lambda\}}(w) d \mathcal{A}(w)=\int_{\mathbb{D}} \mathbb{1}_{\{|F|>\lambda\}}(z)\left|h^{\prime}(z)\right|^{2} d \mathcal{A}(z) \\
& \leq b^{2} \int_{\mathbb{D}} \mathbb{1}_{\{|F|>\lambda\}}(z) d \mathcal{A}(z)=b^{2} \mathcal{A}(\{|F|>\lambda\}) \leq b^{2} \frac{C_{2}}{\lambda^{4}}|f(c)|^{4} .
\end{aligned}
$$

We implicitly used the fact that $F$ maps $\mathbb{D}$ to $G$ and that $|F(0)|=|f(c)|$, so we were allowed to use the previous lemma. Moreover, we have

$$
\mathcal{A}(\Omega)=\int_{\Omega} d \mathcal{A}(w)=\int_{\mathbb{D}}\left|h^{\prime}(z)\right|^{2} d \mathcal{A}(z) \geq a^{2} \mathcal{A}(\mathbb{D})=a^{2},
$$

so that finally

$$
\mathcal{A}(\{|f|>\lambda\}) \leq C_{2} \frac{b^{2}}{a^{2}} \frac{1}{\lambda^{4}} \mathcal{A}(\Omega)|f(c)|^{4} \stackrel{\text { def }}{=} \frac{C}{\lambda^{4}} \mathcal{A}(\Omega)|f(c)|^{4} .
$$

This ends the proof of the first part of Lemma 3.6 for $\Omega$, with $C=C_{2} b^{2} / a^{2}$.

Finally, one has

$$
\begin{aligned}
u(c) & =(u \circ h)(0)=\int_{\mathbb{D}}(u \circ h)(z) d \mathcal{A}(z) \\
& \geq \frac{1}{b^{2}} \int_{\mathbb{D}} u(h(z))\left|h^{\prime}(z)\right|^{2} d \mathcal{A}(z)=\frac{1}{b^{2}} \int_{\Omega} u(w) d \mathcal{A}(w),
\end{aligned}
$$

which gives the right-hand side of (3.7), since $\mathcal{A}(\Omega) \geq a^{2}$. The left-hand side is proved in the same way, since $\mathcal{A}(\Omega) \leq b^{2}$.

The last lemma is of a different kind.

Lemma 3.7. For every analytic function $f: D\left(z_{0}, r\right) \rightarrow G$, one has

$$
\mathcal{A}\left(\left\{\mathfrak{R e} f>\mathfrak{R e} f\left(z_{0}\right) / 2\right\}\right) \geq \frac{1}{8 K_{2}^{2}} \mathcal{A}\left(D\left(z_{0}, r\right)\right),
$$

where $K_{2}$ is the constant given by Lemma 3.5.

Proof. Recall Paley-Zygmund's inequality (see [12, Proposition III.3), usually stated in probabilistic language: for any positive random variable $X$ on some probability space, one has, for $0<a<1$,

$$
\mathbb{P}(X>a \mathbb{E}(X)) \geq(1-a)^{2} \frac{[\mathbb{E}(X)]^{2}}{\mathbb{E}\left(X^{2}\right)},
$$

where $\mathbb{E}$ stands for expectation.

For our problem, we will take as probability space the disk $D\left(z_{0}, r\right)$ equipped with the probability measure $d \mathcal{A} / r^{2}$, and as a random variable $X=\mathfrak{R e} f:=u$. We get from (3.8) that, since $u\left(z_{0}\right)=\mathbb{E}(X)$ by the mean value property of harmonic functions,

$$
\mathcal{A}\left(\left\{u>a u\left(z_{0}\right)\right\}\right) \geq(1-a)^{2} \frac{\left[u\left(z_{0}\right)\right]^{2}}{\mathbb{E}\left(u^{2}\right)} r^{2} .
$$


Now, observe that $\sqrt{2} \mathfrak{R e} w \geq|w|$ when $w \in G$. Hence the function

$$
g(z)=f\left(r z+z_{0}\right) /\left[\sqrt{2} u\left(z_{0}\right)\right]
$$

which maps $\mathbb{D}$ into $G$, satisfies $|g(0)| \leq 1$, and Lemma 3.5 gives $\mathbb{E}\left(u^{2}\right) \leq \mathbb{E}\left(|f|^{2}\right)=$ $\|f\|_{2}^{2} \leq 2 K_{2}^{2}\left[u\left(z_{0}\right)\right]^{2}$. We get

$$
\mathcal{A}\left(\left\{u>a u\left(z_{0}\right)\right\}\right) \geq \frac{(1-a)^{2}}{2 K_{2}^{2}} r^{2},
$$

which gives the desired result by taking $a=1 / 2$.

3.1.2. Proof of Theorem 3.3. For technical reasons, we are going to work with functions with range in the set $G$. Proving Theorem 3.3 amounts to proving:

Proposition 3.8. There exist constants $K^{\prime}>0, \alpha_{1}>0$ and $\lambda_{1}>1$ such that every analytic function $f: \mathbb{D} \rightarrow G$ with $|f(0)| \leq \alpha_{1}$ satisfies, for $\lambda \geq \lambda_{1}$,

$$
\mathcal{A}(\{|f|>\lambda\}) \leq \frac{K^{\prime}}{\lambda^{4}} \mathcal{A}(\{|f|>1\}) .
$$

It will be useful to note that $\sqrt{2} \mathfrak{R e} w \geq|w|$ when $w \in G$.

Proof. The idea of the proof is to split $F_{\lambda}=\{|f|>\lambda\}$ (actually, $F_{\lambda}$ will be conditioned by the more regular set $\left\{M_{d} f>1\right\}$ ) into parts in which we shall be able to apply Lemma 3.6. In order to construct these parts, we shall use a CalderónZygmund type decomposition adapted to the geometry of the unit disk. We are going to recall the principle of this decomposition for the convenience of the reader.

Before that, we have to say that we shall begin to work not with the function $f$ as in the statement of Proposition 3.8, but with this function multiplied by a constant (as we shall specify at the end of the proof). Nevertheless, we shall denote this new function by $f$.

Now, remark that for $f: \mathbb{D} \rightarrow G$ with $|f(0)| \leq \alpha<1$, one has

$$
|f(z)|>1 \quad \Longrightarrow \quad|z|>\beta
$$

for $\beta=\left(1-\alpha^{2}\right) /\left(1+\alpha^{2}\right)$. In fact, setting $a=f(0)$, the function $g=\frac{f^{2}-a^{2}}{f^{2}+\bar{a}^{2}}$ maps $\mathbb{D}$ into itself and vanishes at 0 . By Schwarz's lemma, $|z| \leq \beta$ implies $|g(z)| \leq \beta$, and since $f^{2}=\frac{\bar{a}^{2} g+a^{2}}{1-g}$, we get

$$
|f(z)|^{2} \leq \frac{|a|^{2} \beta+|a|^{2}}{1-\beta}=\frac{|a|^{2}}{\alpha^{2}} \leq 1 .
$$

The goal of the remark (3.9) is that in order to make the Calderón-Zygmund decomposition, we have to avoid the center 0 of $\mathbb{D}$, which is not covered by the sets $S \in \mathcal{S}$ defined below.

For convenience, we shall take $\beta=1 / 2$ (i.e. $\alpha=1 / \sqrt{3}$ ), so we shall only be concerned with the annulus $\Gamma=\{z \in \mathbb{D} ; 1 / 2 \leq|z|<1\}$.

In order to perform the Calderón-Zygmund decomposition, we have to make a splitting of the annulus $\Gamma$. For that, consider the rectangle

$$
R_{0}=\{x+i y ; \log (1 / 2) \leq x<0 \text { and } 0 \leq y<2 \pi\}
$$


and the family $\mathcal{R}=\bigcup_{n \in \mathbb{N}} \mathcal{R}_{n}$ of all dyadic half-open rectangles, where $\mathcal{R}_{n}$ is the family of sets

$$
R=\left\{x+i y \in \mathbb{C} ; x_{j} \leq x<x_{j+1} \quad \text { and } \quad y_{k} \leq y<y_{k+1}\right\},
$$

where $x_{j}=\left(1-j 2^{-n}\right) \log (1 / 2)$ and $y_{k}=2 k \pi / 2^{n}, 0 \leq j, k \leq 2^{n}-1, n \geq 0$.

We now map the annulus $\Gamma$ onto the unit disk $\mathbb{D}$, using the exponential map $x+i y \mapsto z=\mathrm{e}^{x+i y}$. We get a family $\mathcal{S}=\bigcup_{n \in \mathbb{N}} \mathcal{S}_{n}$.

Note that the jacobian $\mathrm{e}^{2 x}$ of the transformation is between $\beta^{2}=1 / 4$ and 1 ; hence the area of a set in $\mathcal{S}_{n-1}$ is less than 16 times that of a set in $\mathcal{S}_{n}$.

Now recall the Calderón-Zygmund decomposition. Consider the conditional expectation $\mathbb{E}_{n}|f|$ of $|f|$, given the $\sigma$-algebra generated by $\mathcal{S}_{n}$; namely,

$$
\left(\mathbb{E}_{n}|f|\right)(z)=\sum_{S \in \mathcal{S}_{n}}\left(\frac{1}{\mathcal{A}(S)} \int_{S}|f| d \mathcal{A}\right) \mathbb{1}_{S}(z) .
$$

The dyadic maximal function $M_{d} f$ is defined by

$$
M_{d} f(z)=\sup _{n}\left(\mathbb{E}_{n}|f|\right)(z) .
$$

Note that

$$
M_{d} f(z)=\sup _{S_{z}} \frac{1}{\mathcal{A}\left(S_{z}\right)} \int_{S_{z}}|f| d \mathcal{A},
$$

where the supremum is taken over all $S_{z} \in \mathcal{S}$ containing $z$.

By Lebesgue's differentiation theorem (or by the martingale convergence theorem), we know that $\left(\mathbb{E}_{n}|f|\right)(z)$ converges to $|f(z)|, \mathcal{A}$-almost everywhere, so $M_{d} f(z)$ $\leq 1$ implies $|f(z)| \leq 1$ for almost all $z \in \mathbb{D}$, and we can write

$$
F_{1}=\{|f|>1\} \subseteq\left\{M_{d} f>1\right\} \cup N,
$$

where $N$ is a negligible set. Hence $F_{\lambda} \subseteq\left\{M_{d} f>1\right\} \cup N$ for $\lambda \geq 1$. Now, the set $\left\{M_{d} f>1\right\}$ can be decomposed in a disjoint union $\left\{M_{d} f>1\right\}=\bigsqcup_{n} E_{n}$, where

$$
E_{n}=\left\{z \in \mathbb{D} ;\left(\mathbb{E}_{n}|f|\right)(z)>1 \text { and }\left(\mathbb{E}_{j}|f|\right)(z) \leq 1 \text { if } j<n\right\} .
$$

Since $\mathbb{E}_{n}|f|$ is constant on the sets $S \in \mathcal{S}_{n}$, each $E_{n}$ can in its turn be decomposed into a disjoint union $E_{n}=\bigsqcup_{k} S_{n, k}$, where $S_{n, k} \in \mathcal{S}_{n}$.

By definition, for $z \in E_{n}$, one has $\left(\mathbb{E}_{n}|f|\right)(z) \geq 1$, and hence

$$
\frac{1}{\mathcal{A}\left(S_{n, k}\right)} \int_{S_{n, k}}|f| d \mathcal{A} \geq 1 \quad \text { for } z \in S_{n, k} .
$$

But, on the other hand, $\left(\mathbb{E}_{n-1}|f|\right)(z) \leq 1$, and we have, if $z \in S_{n, k}$,

$$
\begin{aligned}
\left(\mathbb{E}_{n}|f|\right)(z) & =\frac{1}{\mathcal{A}\left(S_{n, k}\right)} \int_{S_{n, k}}|f| d \mathcal{A} \\
& \leq \frac{1}{\mathcal{A}\left(S_{n, k}\right)} \int_{S_{n-1, j}}|f| d \mathcal{A} \leq 16 \frac{1}{\mathcal{A}\left(S_{n-1, j}\right)} \int_{S_{n-1, j}}|f| d \mathcal{A} \leq 16,
\end{aligned}
$$

where $S_{n-1, j}$ is the set of rank $(n-1)$ containing $S_{n, k}$.

Finally, reindexing the sets $S_{k, n}$, we can write $\left\{M_{d} f>1\right\}$ as a disjoint union

$$
\left\{M_{d} f>1\right\}=\bigsqcup_{l \geq 1} S_{l},
$$




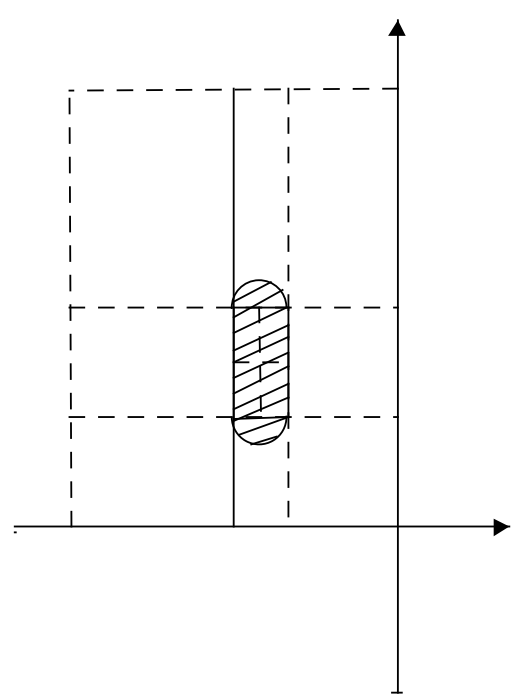

Figure 1. Round set $\hat{R}_{l}$

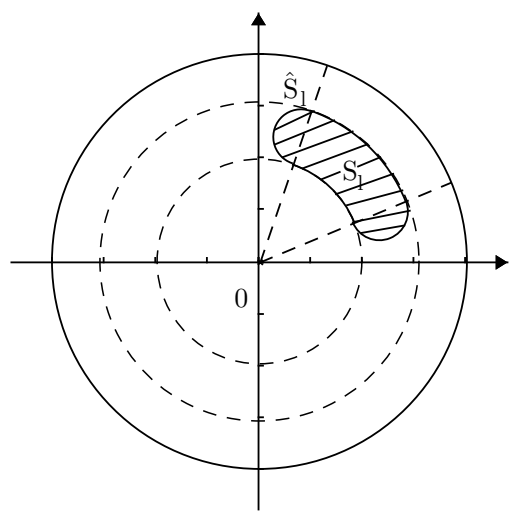

Figure 2. Round set $\hat{S}_{l}$

for which

$$
1 \leq \frac{1}{\mathcal{A}\left(S_{l}\right)} \int_{S_{l}}|f| d \mathcal{A} \leq 16
$$

Equations (3.10), (3.11) and (3.12) define the Calderón-Zygmund decomposition of the function $f$.

In order to apply Lemma 3.6. we have to control (from above and from below) the values of $|f|$ at the "center" of the sets $S_{l}$. One might think of doing this by using (3.12), but it is not always possible (for example, the function with positive real part $1 / z^{2}$ is not integrable on the square of vertices $\left.0,1+i, 1-i, 2\right)$. Nevertheless, since our function is holomorphic on a domain larger than $S_{l}$, we may enlarge $S_{l}$ in order to use (3.12).

Let $R_{l} \in \mathcal{R}$ be the rectangle which is mapped onto $S_{l}$ by the exponential, and let us round $R_{l}$ in $\hat{R}_{l}$ by adding half-disks, as indicated in Figure 1 Let $\hat{S}_{l}=\exp \left(\hat{R}_{l}\right)$.

It is essential, when using Lemma 3.6 for $\Omega=\hat{S}_{l}$, that the constant $C$ given by this lemma does not depend on $l$. This will be checked in the following way. The sets $\hat{R}_{l}$ can be performed by making similarities from $\hat{R}_{0}$, where

$$
R_{0}=\{x+i y ; \log (1 / 2) \leq x<0 \text { and } 0 \leq y<2 \pi\} .
$$

The boundary of $\hat{R}_{0}$ is $\mathcal{C}^{1}$, and it follows from [16], Theorem 3.5, for example, that $\hat{R}_{0}$ is conformally and bi-Lipschitz equivalent to $\mathbb{D}$; therefore, we are able to apply Lemma3.6 (with $c=-\frac{1}{2} \log 2+i \pi$ ). But if $T_{l}\left(\hat{R}_{0}\right)=\hat{R}_{l}$ with $T_{l}(z)=\alpha_{l} z+\beta_{l}$, $\alpha_{l} \neq 0$, we have, if $h_{l}=T_{l} \circ h,\left|h_{l}{ }^{\prime}\right|=\left|\alpha_{l}\right|\left|h^{\prime}\right| \leq\left|\alpha_{l}\right| b$, as well as $\left|h_{l}{ }^{\prime}\right| \geq\left|\alpha_{l}\right| a$, so that $a$ and $b$ are respectively changed into $a_{l}=\left|\alpha_{l}\right| a$ and $b_{l}=\left|\alpha_{l}\right| b$, and the quotient $b_{l} / a_{l}=b / a$ remains unchanged. Now, $\hat{S}_{l}=\exp \left(\hat{R}_{l}\right)$ and $1 / 2 \leq|\exp (x+i y)|=$ $\mathrm{e}^{x} \leq 1$ for $x+i y \in \hat{R}_{0}$. Hence the constant $C=C_{2} b^{2} / a^{2}$ of Lemma 3.6 for $\Omega=\hat{R}_{0}$ becomes $C \leq 4 C_{2} b^{2} / a^{2}$ for $\Omega=\hat{S}_{l}$. 


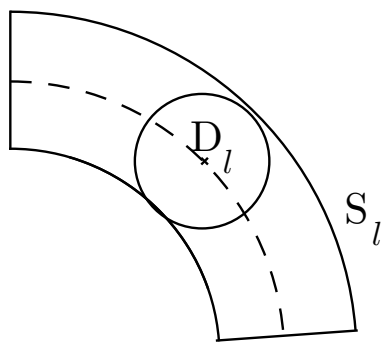

FiguRE 3. Disk $D_{l}$

Therefore, one has, for every $l \geq 1$, if $\gamma_{l}$ is the center (defined in an obvious way) of $S_{l}$ (and hence of $\hat{S}_{l}$ ),

$$
\mathcal{A}\left(\left\{z \in \hat{S}_{l} ;|f(z)|>\lambda\right\}\right) \leq \frac{C}{\lambda^{4}}\left|f\left(\gamma_{l}\right)\right|^{4} \mathcal{A}\left(\hat{S}_{l}\right) .
$$

Now, let $D_{l}$ be the greatest open disk with center $\gamma_{l}$ and contained in $S_{l}$ (see Figure 3). We have, by the last part of Lemma 3.6.

$$
\left|f\left(\gamma_{l}\right)\right| \leq \frac{C}{\mathcal{A}\left(D_{l}\right)} \int_{D_{l}}|f| d \mathcal{A} \leq \frac{64 C}{\mathcal{A}\left(S_{l}\right)} \int_{S_{l}}|f| d \mathcal{A} \leq 2^{12} C .
$$

Using the fact that $\mathcal{A}\left(\hat{S}_{l}\right) \leq 4 \mathcal{A}\left(S_{l}\right)$ and that $F_{\lambda} \subseteq\left\{M_{d} f>1\right\} \cup N$ when $\lambda \geq 1$, we get

$$
\begin{aligned}
\mathcal{A}\left(F_{\lambda}\right) & =\mathcal{A}\left(F_{\lambda} \cap\left\{M_{d} f>1\right\}\right) \leq \sum_{l \geq 1} \mathcal{A}\left(\left\{z \in \hat{S}_{l} ;|f(z)|>\lambda\right\}\right) \\
& \leq \frac{C}{\lambda^{4}}\left(2^{12} C\right)^{4} \sum_{l \geq 1} \mathcal{A}\left(\hat{S}_{l}\right) \leq \frac{2^{52} C^{5}}{\lambda^{4}} \sum_{l \geq 1} \mathcal{A}\left(S_{l}\right) \\
& =\frac{2^{52} C^{5}}{\lambda^{4}} \mathcal{A}\left(\left\{M_{d} f>1\right\}\right) .
\end{aligned}
$$

It remains to control $\mathcal{A}\left(\left\{M_{d} f>1\right\}\right)$ by $\mathcal{A}(\{|f|>\delta\})$, for some numerical $\delta>0$.

For that, we shall use Lemma 3.7. By harmonicity and Lemma 3.6, one has, with $u=\mathfrak{R e} f$,

$$
\begin{aligned}
u\left(\gamma_{l}\right) & \geq \frac{1 / C}{\mathcal{A}\left(\hat{S}_{l}\right)} \int_{\hat{S}_{l}} u d \mathcal{A} \geq \frac{1}{16 C} \frac{1}{\mathcal{A}\left(S_{l}\right)} \int_{S_{l}} u d \mathcal{A} \\
& \geq \frac{1}{16 \sqrt{2} C} \frac{1}{\mathcal{A}\left(S_{l}\right)} \int_{S_{l}}|f| d \mathcal{A} \geq \frac{1}{16 \sqrt{2} C} .
\end{aligned}
$$

We now apply Lemma 3.7 and obtain

$$
\begin{aligned}
\mathcal{A}\left(\{|f|>1 / 64 C\} \cap D_{l}\right) & \geq \mathcal{A}\left(\{u>1 / 32 \sqrt{2} C\} \cap D_{l}\right) \geq \mathcal{A}\left(\left\{u>u\left(\gamma_{l}\right) / 2\right\} \cap D_{l}\right) \\
& \geq \frac{1}{8 K_{2}^{2}} \mathcal{A}\left(D_{l}\right) .
\end{aligned}
$$

Hence we obtain

$$
\mathcal{A}\left(\left\{M_{d} f>1\right\}\right)=\sum_{l \geq 1} \mathcal{A}\left(S_{l}\right) \leq 16 \sum_{l \geq 1} \mathcal{A}\left(D_{l}\right) \leq 128 K_{2}^{2} \mathcal{A}(\{|f|>1 / 64 C\}) .
$$


The proof of Theorem 3.3 is now finished, because (3.15) and (3.16) give, for $\lambda \geq 1$,

$$
\mathcal{A}(\{|f|>\lambda\}) \leq \frac{2^{38} C^{5} K_{2}^{2}}{\lambda^{4}} \mathcal{A}(\{|f|>1 / 64 C\}),
$$

and if $f$ is as in the statement of Proposition 3.8, we can apply (3.17) to $f_{1}=64 C f$ when $\left|f_{1}(0)\right| \leq 1 / \sqrt{3}$, and we get, for $\lambda \geq \lambda_{1}=64 C$,

$$
\mathcal{A}(\{|f|>\lambda\}) \leq \frac{2^{38}(64 C)^{4} C^{5} K_{2}^{2}}{\lambda^{4}} \mathcal{A}(\{|f|>1\})
$$

when $|f(0)| \leq 1 /(64 C \sqrt{3})$.

3.2. Nevanlinna counting function. The Nevanlinna counting function is defined, for every analytic function $\varphi: \mathbb{D} \rightarrow \mathbb{D}$ and for every $w \in \varphi(\mathbb{D}) \backslash\{\varphi(0)\}$, by

$$
N_{\varphi}(w)=\sum_{\varphi(z)=w} \log \frac{1}{|z|},
$$

where each term $\log \frac{1}{|z|}$ is repeated according to the multiplicity of $z$, and by $N_{\varphi}(w)=0$ for the other $w \in \mathbb{D}$.

Recall (see 9]) that if $m$ is the normalized Lebesgue measure on $\mathbb{T}$, then the Carleson function of $\varphi$ is the Carleson function of the pull-back measure $m_{\varphi}$ of $m$ by $\varphi$. We proved in [9] (Theorem 3.1 and Theorem 3.7) that the behaviour of $N_{\varphi}$ is equivalent to that of the Carleson function $\rho_{\varphi}$ in the following way.

Theorem 3.9 (9]). There exist two universal constants $C, c>1$ such that, for every analytic self-map $\varphi: \mathbb{D} \rightarrow \mathbb{D}$, one has

$$
\sup _{w \in W(\xi, h) \cap \mathbb{D}} N_{\varphi}(w) \leq C m_{\varphi}[W(\xi, c h)]
$$

and

$$
m_{\varphi}(W(\xi, h)) \leq C \frac{1}{\mathcal{A}(W(\xi, c h))} \int_{W(\xi, c h)} N_{\varphi}(z) d \mathcal{A}(z)
$$

for $0<h<1$ small enough.

We are going to deduce from Theorem 3.9 the same result in the 2-dimensional case. The Nevanlinna counting function of order 2 is defined (see [18, § 6.2) for $w \in \mathbb{D} \backslash\{\varphi(0)\}$ by

$$
N_{\varphi, 2}(w)=\sum_{\varphi(z)=w}(\log (1 /|z|))^{2},
$$

where each preimage $z$ of $w$ appears as often as its multiplicity. The partial Nevanlinna counting function is defined for $0<r \leq 1$ by

$$
N_{\varphi}(r, w)=\sum_{\varphi(z)=w,|z|<r} \log (r /|z|),
$$

and we have ([18, Proposition 6.6, where a misprint occurs)

$$
N_{\varphi, 2}(w)=2 \int_{0}^{1} N_{\varphi}(r, w) \frac{d r}{r} .
$$

One has the following theorem. 
Theorem 3.10. There exists a universal constant $C>1$, such that for every analytic self-map $\varphi: \mathbb{D} \rightarrow \mathbb{D}$, one has

$$
(1 / C) \rho_{\varphi, 2}(h / C) \leq \sup _{|w| \geq 1-h} N_{\varphi, 2}(w) \leq C \rho_{\varphi, 2}(C h),
$$

for $0<h<1$ small enough.

Proof. Let $w_{0}=\varphi(0)$ and set

$$
u(z)=\frac{w_{0}-\varphi(z)}{1-\bar{w}_{0} \varphi(z)} .
$$

Since $u(0)=0$, Schwarz's lemma gives $|u(z)| \leq|z|$. Hence there is no $z$ with $|z|<t$ such that $\varphi(z)=w$ when $t \leq\left|w_{0}-w\right| /\left|1-\bar{w}_{0} w\right|:=\left|u_{0}(w)\right|$. It follows that (3.22) actually writes

$$
N_{\varphi, 2}(w)=2 \int_{\left|u_{0}(w)\right|}^{1} N_{\varphi}(r, w) \frac{d r}{r} .
$$

1) It follows from (3.24) that

$$
N_{\varphi, 2}(w) \leq \frac{2}{\left|u_{0}(w)\right|^{2}} \int_{0}^{1} N_{\varphi}(r, w) r d r .
$$

But (see [9], Lemma 3.4) $\left|u_{0}(w)\right|=\left|w-w_{0}\right| /\left|1-\bar{w} w_{0}\right|>1 / 3$ when $1-|w|<$ $\left(1-\left|w_{0}\right|\right) / 4$; therefore, for $1-|w|<\left(1-\left|w_{0}\right|\right) / 4$ we have

$$
N_{\varphi, 2}(w) \leq 18 \int_{0}^{1} N_{\varphi}(r, w) r d r .
$$

Now, $N_{\varphi}(r, w)=N_{\varphi_{r}}(w)$, where $\varphi_{r}(z)=\varphi(r z)$, and it follows from (3.19) that, for $w \in W(\xi, h)$ with $\xi \in \mathbb{T}$ and $h>0$ small enough, one has

$$
\begin{aligned}
N_{\varphi, 2}(w) & \leq 18 \int_{0}^{1} C m_{\varphi_{r}}[W(\xi, c h)] r d r \\
& =18 C \int_{0}^{1} m\left(\left\{\mathrm{e}^{i \theta} ; \varphi\left(r \mathrm{e}^{i \theta}\right) \in W(\xi, c h)\right\}\right) r d r \\
& =9 C \mathcal{A}(\{z \in \mathbb{D} ; \varphi(z) \in W(\xi, c h)\}) .
\end{aligned}
$$

2) Conversely, it follows from (3.22) that

$$
N_{\varphi, 2}(w) \geq 2 \int_{0}^{1} N_{\varphi}(r, w) r d r .
$$

Hence

$$
\begin{aligned}
\frac{1}{\mathcal{A}[W(\xi, c h)]} & \int_{W(\xi, c h)} N_{\varphi, 2}(z) d \mathcal{A}(z) \\
& \geq 2 \int_{0}^{1}\left[\frac{1}{\mathcal{A}[W(\xi, c h)]} \int_{W(\xi, c h)} N_{\varphi}(r, z) d \mathcal{A}(z)\right] r d r \\
& \geq \frac{2}{C} \int_{0}^{1} m_{\varphi_{r}}[W(\xi, h)] r d r \\
& =\frac{2}{C} \int_{0}^{1} m\left(\left\{\mathrm{e}^{i \theta} ; \varphi\left(r \mathrm{e}^{i \theta}\right) \in W(\xi, h)\right\}\right) r d r \\
& =\frac{1}{C} \mathcal{A}(\{z \in \mathbb{D} ; \varphi(z) \in W(\xi, h)\}),
\end{aligned}
$$

and that finishes the proof of Theorem 3.10 . 
Remark. Actually, the proof shows that for some constant $C>1$, one has

$$
(1 / C) \mathcal{A}_{\varphi}[W(\xi, h / C)] \leq \sup _{w \in W(\xi, h)} N_{\varphi, 2}(w) \leq C \mathcal{A}_{\varphi}[W(\xi, C h)],
$$

for every $\xi \in \mathbb{T}$ and $0<h<1$ small enough. Since the $\ell_{2}$-norm is less than the $\ell_{1}$-norm, one has $N_{\varphi, 2}(w) \leq\left[N_{\varphi}(w)\right]^{2}$. Hence it follows from Theorem 3.9 and Theorem 3.10 (actually (3.25) ) that for some constant $C>1$, one has

$$
\mathcal{A}(\{z \in \mathbb{D} ; \varphi(z) \in W(\xi, h)\}) \leq C\left[m\left(\left\{u \in \mathbb{T} ; \varphi^{*}(u) \in W(\xi, C h)\right\}\right)\right]^{2},
$$

for every $\xi \in \mathbb{T}$ and every $0<h<1$ small enough, a fact which does not seem easy to prove in a straightforward way. In particular, for some constant $C>0$, one has, for $h>0$ small enough,

$$
\rho_{\varphi, 2}(h) \leq C\left[\rho_{\varphi}(C h)\right]^{2} .
$$

Corollary 3.11. For every analytic self-map $\varphi: \mathbb{D} \rightarrow \mathbb{D}$ and for every Orlicz function $\Psi$, the composition operator $C_{\varphi}: \mathfrak{B}^{\Psi} \rightarrow \mathfrak{B}^{\Psi}$ is compact if and only if

$$
\lim _{h \rightarrow 0} \frac{\Psi^{-1}\left(1 / h^{2}\right)}{\Psi^{-1}\left(1 / \nu_{\varphi, 2}(h)\right)}=0,
$$

where $\nu_{\varphi, 2}(h)=\sup _{|w| \geq 1-h} N_{\varphi, 2}(w)$.

Proof. If $C_{\varphi}$ is compact, Theorem 3.2 gives, for every $A>0$, an $h_{A}>0$ such that for $0<h \leq h_{A}$

$$
\Psi^{-1}\left(1 / h^{2}\right) \leq \frac{1}{A} \Psi^{-1}\left(1 / \rho_{\varphi, 2}(h)\right) .
$$

Then Theorem 3.10 gives

$$
\nu_{\varphi, 2}(h) \leq C \rho_{\varphi, 2}(C h) \leq C / \Psi\left[A \Psi^{-1}\left(1 / C^{2} h^{2}\right)\right],
$$

i.e. $A \Psi^{-1}\left(1 / C^{2} h^{2}\right) \leq \Psi^{-1}\left[C / \nu_{\varphi, 2}(h)\right]$, and then, by concavity, since $C>1$,

$$
\frac{1}{C^{2}} \Psi^{-1}\left(1 / h^{2}\right) \leq \Psi^{-1}\left(1 / C^{2} h^{2}\right) \leq \frac{1}{A} \Psi^{-1}\left(C / \nu_{\varphi, 2}(h)\right) \leq \frac{C}{A} \Psi^{-1}\left(1 / \nu_{\varphi, 2}(h)\right),
$$

which implies (3.27).

The converse follows the same lines.

Corollary 3.12. The compactness of the composition operator $C_{\varphi}: \mathfrak{B}^{\Psi} \rightarrow \mathfrak{B}^{\Psi}$ implies that

$$
\lim _{|z| \rightarrow 1} \frac{\Psi^{-1}\left(1 /[1-|\varphi(z)|]^{2}\right)}{\Psi^{-1}\left(1 /(1-|z|)^{2}\right)}=0 .
$$

This corollary was proved in [7, Theorem 5.7, by a more direct method, and we also showed that the condition is sufficient when $\Psi$ grows fast enough (namely, satisfies the condition $\Delta^{2}$ ). We do not know whether it is not sufficient for some symbol $\varphi$ and some Orlicz function $\Psi$. If one assumes a condition of regularity on $\Psi$, namely the so-called condition $\nabla_{0}$, then condition (3.28) is necessary and sufficient for the compactness of $C_{\varphi}: \mathfrak{B}^{\Psi} \rightarrow \mathfrak{B}^{\Psi}$ ([11], Proposition 2). Nevertheless, without any condition on $\Psi$, it follows easily from Corollary 3.11 that this condition is sufficient when $\varphi$ is boundedly valent (see 9], proof of Theorem 5.3). 
Proof. Since $N_{\varphi, 2}(\varphi(z)) \geq(\log (1 /|z|))^{2} \geq(1-|z|)^{2}$, it follows from Corollary 3.11 that, for every $A>0$, one has

$$
(1-|z|)^{2} \leq \frac{1}{\Psi\left[A \Psi^{-1}\left(1 /(1-|\varphi(z)|)^{2}\right)\right]} ;
$$

that is,

$$
\frac{\Psi^{-1}\left(1 /(1-|\varphi(z)|)^{2}\right)}{\Psi^{-1}\left(1 /(1-|z|)^{2}\right)} \leq 1 / A
$$

and that proves Corollary 3.12 .

\section{Comparison of the COMPACTNESS OF COMPOSITION OPERATORS on Hardy-Orlicz SPACES AND ON Bergman-Orlicz SPACES}

In the classical case $\left(\Psi(x)=x^{p}, 1 \leq p<\infty\right)$, it is known ([14], Theorem 3.5, with Proposition 2.7) that the compactness of $C_{\varphi}: H^{p} \rightarrow H^{p}$ implies the compactness of $C_{\varphi}: \mathfrak{B}^{p} \rightarrow \mathfrak{B}^{p}$. On the other hand, we implicitly proved in [7, Theorem 5.7, that when $\Psi$ grows very fast (namely, satisfies the so-called $\Delta^{2}$ condition), then the compactness of $C_{\varphi}: H^{\Psi} \rightarrow H^{\Psi}$ implies the compactness of $C_{\varphi}: \mathfrak{B}^{\Psi} \rightarrow \mathfrak{B}^{\Psi}$. Let us write why: it is easy to show (see 9 , proof of Theorem 4.3) that the compactness of $C_{\varphi}: H^{\Psi} \rightarrow H^{\Psi}$ implies that $\Psi^{-1}[1 /(1-|\varphi(z)|)] / \Psi^{-1}[1 /(1-|z|)]$ tends to 0 as $|z|$ goes to 1 , and we actually proved in 7 , Theorem 5.7, that when $\Psi \in \Delta^{2}$, this last condition implies the compactness of $C_{\varphi}: \mathfrak{B}^{\Psi} \rightarrow \mathfrak{B}^{\Psi}$. The next proposition gives a condition on $\Psi$ which, though not very satisfactory, includes the cases $\Psi(x)=x^{p}$ and $\Psi \in \Delta^{2}$ and for which compactness on $H^{\Psi}$ implies compactness on $\mathfrak{B}^{\Psi}$.

Proposition 4.1. Assume that the Orlicz function $\Psi$ satisfies the following condition. For every $A>0$, there exist $x_{A}>0$ and $B \geq A$ such that

$$
\Psi\left[A \Psi^{-1}\left(x^{2}\right)\right] \leq\left(\Psi\left[B \Psi^{-1}(x)\right]\right)^{2}
$$

for every $x \geq x_{A}$. Then every analytic map $\varphi: \mathbb{D} \rightarrow \mathbb{D}$ defining a compact composition operator $C_{\varphi}: H^{\Psi} \rightarrow H^{\Psi}$ also defines a compact composition operator $C_{\varphi}: \mathfrak{B}^{\Psi} \rightarrow \mathfrak{B}^{\Psi}$

It is clear that $\Psi$ satisfies (4.1) if $\Psi(x)=x^{p}$. Recall that condition $\Delta^{2}$ means that there exists some $\alpha>1$ such that $[\Psi(t)]^{2} \leq \Psi(\alpha t)$ for $t$ large enough. For such a function, one has $\Psi^{-1}\left(x^{2}\right) \leq \alpha \Psi^{-1}(x)$, and hence $\Psi\left[A \Psi^{-1}\left(x^{2}\right)\right] \leq \Psi\left[\alpha A \Psi^{-1}(x)\right]$, which is $\leq\left(\Psi\left[\alpha A \Psi^{-1}(x)\right]\right)^{2}$ for $x$ large enough, since it tends to infinity. Therefore, (4.1) holds if $\Psi$ has $\Delta^{2}$. Classical examples of Orlicz functions with $\Delta^{2}$ are $\Psi(x)=$ $\mathrm{e}^{x^{q}}-1$, where $q \geq 1$.

Another example, which does not have $\Delta^{2}$ but satisfies (4.1), is

$$
\Psi(x)=\exp \left([\log (x+1)]^{2}\right)-1 .
$$

Proof of Proposition 4.1. One has $N_{\varphi, 2}(w) \leq\left[N_{\varphi}(w)\right]^{2}$, since the $\ell_{2}$-norm is less than the $\ell_{1}$-norm. Let $A>0$ be arbitrary. If $C_{\varphi}$ is compact on $H^{\Psi}$, we know, by [9], Theorem 4.2, that

$$
\sup _{|w| \geq 1-h} N_{\varphi}(w)=o\left(\frac{1}{\Psi\left[B \Psi^{-1}(1 / h)\right]}\right), \quad \text { as } h \rightarrow 0 .
$$


By (4.1), we get

$$
\sup _{|w| \geq 1-h} N_{\varphi, 2}(w)=o\left(\frac{1}{\Psi\left[A \Psi^{-1}\left(1 / h^{2}\right)\right]}\right) .
$$

Corollary 3.11 ensures that $C_{\varphi}$ is compact on $\mathfrak{B}^{\Psi}$.

However, we are going to see that the conclusion of Proposition 4.1 does not hold for an arbitrary Orlicz function by proving the following theorem.

Theorem 4.2. There exist an analytic self-map $\varphi: \mathbb{D} \rightarrow \mathbb{D}$ and an Orlicz function $\Psi$ such that $C_{\varphi}: H^{\Psi} \rightarrow H^{\Psi}$ is compact whereas $C_{\varphi}: \mathfrak{B}^{\Psi} \rightarrow \mathfrak{B}^{\Psi}$ is not compact.

In order to prove it, we shall show and use the following result.

Theorem 4.3. There exists an analytic self-map $\varphi: \mathbb{D} \rightarrow \mathbb{D}$ such that, for some constants $c_{2} \geq \pi$ and $\pi / 4 \geq c_{1}>0$, one has, for some constant $C>0$ and for $h>0$ small enough,

$$
\begin{aligned}
\rho_{\varphi}(h) & \leq C \mathrm{e}^{-c_{1} / h}, \\
\rho_{\varphi, 2}(h) & \geq(1 / C) \mathrm{e}^{-c_{2} / h} .
\end{aligned}
$$

Proof of Theorem 4.2. We know ([7], Theorem 4.18) that $C_{\varphi}: H^{\Psi} \rightarrow H^{\Psi}$ is compact if and only if

$$
\lim _{h \rightarrow 0} \frac{\Psi^{-1}(1 / h)}{\Psi^{-1}\left(1 / \rho_{\varphi}(h)\right)}=0
$$

and (by Theorem 3.2 ) that the compactness of $C_{\varphi}: \mathfrak{B}^{\Psi} \rightarrow \mathfrak{B}^{\Psi}$ is equivalent to

$$
\lim _{h \rightarrow 0} \frac{\Psi^{-1}\left(1 / h^{2}\right)}{\Psi^{-1}\left(1 / \rho_{\varphi, 2}(h)\right)}=0 .
$$

Hence, it suffices to construct an Orlicz function $\Psi$ such that

$$
\lim _{x \rightarrow \infty} \frac{\Psi^{-1}(x)}{\Psi^{-1}\left(\mathrm{e}^{c_{1} x}\right)}=0
$$

and

$$
\limsup _{x \rightarrow \infty} \frac{\Psi^{-1}\left(x^{2}\right)}{\Psi^{-1}\left(\mathrm{e}^{c_{2} x}\right)}>0 .
$$

We shall actually construct an increasing concave function $f:[0, \infty) \rightarrow \mathbb{R}_{+}$such that $f(0)=0$ and $f(\infty)=\infty$, which satisfies (4.6) and (4.7) (with $f$ instead of $\left.\Psi^{-1}\right)$, and we shall then take $\Psi=f^{-1}$.

1) For that, we set $\alpha_{0}=0$ and we define an increasing sequence of positive numbers $\alpha_{1}=1, \alpha_{2}, \ldots$ by

$$
\alpha_{n+1}=\mathrm{e}^{c_{1} \alpha_{n}}, \quad n \geq 0,
$$

and we take $f$ affine on each interval $\left[\alpha_{n}, \alpha_{n+1}\right], n \geq 0$. More precisely, we set

$$
f(t)=A_{n} t+B_{n}, \quad \text { for } \quad \alpha_{n-1} \leq t \leq \alpha_{n}, \quad n \geq 1,
$$

where $A_{1}=1, B_{1}=0$, and for $n \geq 1$

$$
B_{n+1}-B_{n}=\left(A_{n}-A_{n+1}\right) \alpha_{n}
$$


and for $n \geq 0$

$$
\frac{B_{n+1}}{A_{n+1}}=\frac{1}{2}\left(\mathrm{e}^{c_{2} \sqrt{\alpha_{n}}}-3 \alpha_{n}\right) .
$$

Condition (4.10) ensures that $f$ is continuous. It is clear that $f$ is increasing and that $f(\infty)=\infty$.

Now, since $c_{2}>\sqrt{6}$, the function $u$ defined by $u(x)=\mathrm{e}^{c_{2} x}-3 x^{2}$ is positive and increasing for $x>0$. Hence, if one sets

$$
\beta_{n}=\left(\mathrm{e}^{c_{2} \sqrt{\alpha_{n}}}-3 \alpha_{n}\right) / 2, \quad n \geq 0,
$$

$\left(\beta_{n}\right)_{n}$ is an increasing sequence of positive numbers. But $\beta_{n}=B_{n+1} / A_{n+1}$; hence (4.10) gives for $n \geq 1$,

$$
A_{n}=\frac{\alpha_{n}+\beta_{n}}{\alpha_{n}+\beta_{n-1}} A_{n+1}
$$

Since $\beta_{n}>\beta_{n-1}$, it follows that $A_{n}>A_{n+1}$, and so the function $f$ is concave.

2) For $n$ large enough, one has $\alpha_{n}<\mathrm{e}^{c_{2} \sqrt{\alpha_{n}}}<\mathrm{e}^{c_{1} \alpha_{n}}=\alpha_{n+1}$; hence, for these $n$,

$$
\frac{f\left(\alpha_{n}\right)}{f\left(\mathrm{e}^{c_{2} \sqrt{\alpha_{n}}}\right)}=\frac{A_{n+1} \alpha_{n}+B_{n+1}}{A_{n+1} \mathrm{e}^{c_{2} \sqrt{\alpha_{n}}}+B_{n+1}}=\frac{\alpha_{n}+\beta_{n}}{\mathrm{e}^{c_{2} \sqrt{\alpha_{n}}}+\beta_{n}}=\frac{1}{3},
$$

and it follows that

$$
\limsup _{x \rightarrow \infty} \frac{f\left(x^{2}\right)}{f\left(\mathrm{e}^{c_{2} x}\right)} \geq \frac{1}{3}
$$

Condition (4.7) is satisfied.

3) It remains to check condition (4.6).

For that, we shall fix a number $M>c_{2} / c_{1}$ and take $n_{0}$ large enough to have $\alpha_{n-1}<M \sqrt{\alpha_{n}}<\alpha_{n}$ for $n \geq n_{0}$.

Let $x_{0}$ be such that $x \geq x_{0}$ if and only if $\alpha_{n-1} \leq x<\alpha_{n}$ with $n \geq n_{0}$. Choose such an $x$. We have

$$
\alpha_{n}=\mathrm{e}^{c_{1} \alpha_{n-1}} \leq \mathrm{e}^{c_{1} x} \leq \mathrm{e}^{c_{1} \alpha_{n}}=\alpha_{n+1} .
$$

We shall separate two cases. For convenience, we set $\varepsilon_{n}=1 / \beta_{n}$.

a) Case 1: $\alpha_{n-1} \leq x<M \sqrt{\alpha_{n}}$. Then

$$
\begin{aligned}
\frac{f(x)}{f\left(\mathrm{e}^{c_{1} x}\right)} & =\frac{A_{n} x+B_{n}}{A_{n+1} \mathrm{e}^{c_{1} x}+B_{n+1}} \leq \frac{A_{n} M \sqrt{\alpha_{n}}+B_{n}}{A_{n+1} \alpha_{n}+B_{n+1}} \\
& =\frac{A_{n} M \sqrt{\alpha_{n}}+B_{n}}{A_{n} \alpha_{n}+B_{n}}, \quad \text { by (4.10) } \\
& =\frac{\varepsilon_{n-1} M \sqrt{\alpha_{n}}+1}{\varepsilon_{n-1} \alpha_{n}+1} \sim \frac{M}{\sqrt{\alpha_{n}}},
\end{aligned}
$$

since

$$
\varepsilon_{n-1} \sqrt{\alpha_{n}}=2 \frac{\mathrm{e}^{c_{1} \alpha_{n-1} / 2}}{\mathrm{e}^{c_{2} \sqrt{\alpha_{n-1}}}-3 \alpha_{n-1}} \sim 2 \exp \left(\frac{c_{1}}{2} \alpha_{n-1}-c_{2} \sqrt{\alpha_{n-1}}\right) \underset{n \rightarrow \infty}{\longrightarrow}+\infty .
$$


b) Case 2: $M \sqrt{\alpha_{n}} \leq x<\alpha_{n}$. Then

$$
\begin{aligned}
\frac{f(x)}{f\left(\mathrm{e}^{c_{1} x}\right)} \leq & \frac{f\left(\alpha_{n}\right)}{f\left(\mathrm{e}^{c_{1} M \sqrt{\alpha_{n}}}\right)}=\frac{A_{n+1} \alpha_{n}+B_{n+1}}{A_{n+1} \mathrm{e}^{c_{1} M \sqrt{\alpha_{n}}}+B_{n+1}}=\frac{\varepsilon_{n} \alpha_{n}+1}{\varepsilon_{n} \mathrm{e}^{c_{1} M \sqrt{\alpha_{n}}}+1} \\
= & \frac{\frac{2 \alpha_{n}}{\mathrm{e}^{c_{2} \sqrt{\alpha_{n}}}-3 \alpha_{n}}+1}{2 \frac{\mathrm{e}^{c_{1} M \sqrt{\alpha_{n}}}}{\mathrm{e}^{c_{2} \sqrt{\alpha_{n}}}-3 \alpha_{n}}+1} \sim \exp \left(\left(c_{2}-M c_{1}\right) \sqrt{\alpha_{n}}\right) \underset{n \rightarrow \infty}{\longrightarrow} 0,
\end{aligned}
$$

since $M c_{1}>c_{2}$.

Putting the two cases together, we obtain $\lim _{x \rightarrow+\infty} \frac{f(x)}{f\left(\mathrm{e}^{c_{1} x}\right)}=0$, so (4.6) is satisfied, and Theorem 4.2 is fully proved.

Proof of Theorem 4.3. The analytic map $\varphi$ will be a conformal mapping from $\mathbb{D}$ to the domain $G$, edged by three circular arcs of radii $1 / 2$, and which is represented in Figure 4.

More precisely, let $G_{0}=\mathbb{D} \cap\{\Re \mathrm{e} z>0\}$ and let $f: \mathbb{D} \rightarrow G_{0}$ be the conformal map such that

$$
f(-1)=0 ; \quad f(1)=1 ; \quad f(i)=i ; \quad f(-i)=-i .
$$

We successively define $\varphi_{1}(z)=\log f(z)$, which maps $\mathbb{D}$ onto the half-strip $\{\Re$ e $w<$ $0,|\Im \mathrm{Im} w|<\pi / 2\}, \varphi_{2}(z)=-\frac{2}{\pi} \varphi_{1}(z)+1, \varphi_{3}(z)=\frac{1}{\varphi_{2}(z)}$, and finally $\varphi(z)=\varphi_{3}(z)-1$.

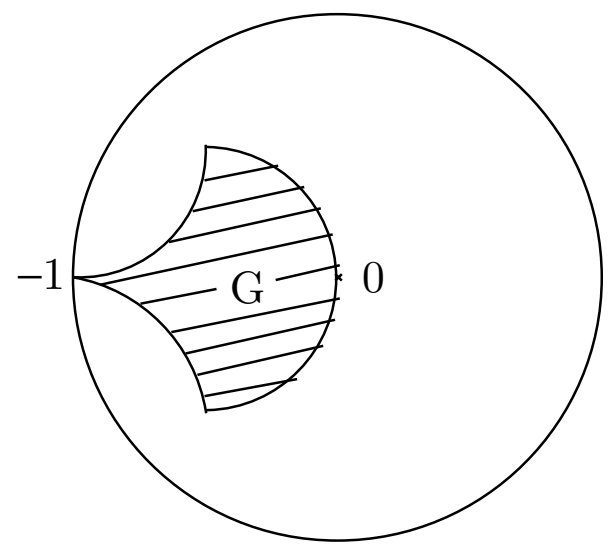

Figure 4. Domain $G$

1) When $W(\xi, h) \cap G \neq \emptyset$, we must have $W(\xi, h) \cap G \subseteq S(-1,2 h)$, for $h$ small enough. Hence

$$
\begin{aligned}
\rho_{\varphi}(h) & \leq m\left(\left\{z \in \mathbb{T} ;\left|\varphi_{3}(z)\right|<2 h\right\}\right)=m\left(\left\{z \in \mathbb{T} ;\left|\varphi_{2}(z)\right|>1 / 2 h\right\}\right) \\
& \leq m\left(\left\{z \in \mathbb{T} ; \mathfrak{R e} \varphi_{2}(z)>1 / 2 h-1\right\}\right), \quad \text { since }\left|\mathfrak{I m} \varphi_{2}(z)\right|<1 \\
& =m\left(\left\{z \in \mathbb{T} ; \mathfrak{R e} \varphi_{1}(z)<\pi-\pi /(4 h)\right\}\right) \\
& =m\left(\left\{z \in \mathbb{T} ;|f(z)|<\mathrm{e}^{\pi} \mathrm{e}^{-\pi / 4 h}\right\}\right) \\
& \leq K\left|\left\{t \in[-1,1] ;|i t|<\mathrm{e}^{\pi} \mathrm{e}^{-\pi / 4 h}\right\}\right|=2 K \mathrm{e}^{\pi} \mathrm{e}^{-\pi / 4 h},
\end{aligned}
$$

for small $h>0$. 
2) On the other hand, $S(-1, h) \cap G \subseteq W(-1, h)$, so

$$
\begin{aligned}
\rho_{\varphi, 2}(h) & \geq \mathcal{A}(\{z \in \mathbb{D} ; \varphi(z) \in W(-1, h)\}) \\
& \geq \mathcal{A}\left(\left\{z \in \mathbb{D} ;\left|\varphi_{3}(z)\right|<h\right\}\right)=\mathcal{A}\left(\left\{z \in \mathbb{D} ;\left|\varphi_{2}(z)\right|>1 / h\right\}\right) \\
& \geq \mathcal{A}\left(\left\{z \in \mathbb{D} ; \mathfrak{R e} \varphi_{1}(z)<\frac{\pi}{2}\left(1-\frac{1}{h}\right)\right\}\right) \\
& =\mathcal{A}\left(\left\{z \in \mathbb{D} ;|f(z)|<\mathrm{e}^{\pi / 2} \mathrm{e}^{-\pi / 2 h}\right\}\right) \\
& \geq c \mathcal{A}\left(\left\{z \in \mathbb{D} ;|z|<\mathrm{e}^{\pi / 2} \mathrm{e}^{-\pi / 2 h}\right\}\right)=c^{\prime} \mathrm{e}^{\pi} \mathrm{e}^{-\pi / h},
\end{aligned}
$$

for $h>0$ small enough.

The proof of Theorem 4.3 is complete.

Remark. In Theorem 4.3, we have in particular $\rho_{\varphi}(h)=O\left(h^{\alpha}\right)$ (i.e. $m_{\varphi}$ is an $\alpha$ Carleson measure) for every $\alpha \geq 1$. Hence ([8, Proposition 3.2) the composition operator $C_{\varphi}: H^{2} \rightarrow H^{2}$ is in all the Schatten classes $S_{p}\left(H^{2}\right), p>0$.

Remark. The compactness of $C_{\varphi}: H^{\Psi} \rightarrow H^{\Psi}$ implies the compactness of $C_{\varphi}: \mathfrak{B}^{\Psi^{2}} \rightarrow$ $\mathfrak{B}^{\Psi^{2}}$ since, if one sets $\tilde{\Psi}(x)=[\Psi(x)]^{2}$, then $\tilde{\Psi}^{-1}\left(t^{2}\right)=\Psi^{-1}(t)$, and hence

$$
\tilde{\Psi}^{-1}\left(1 / h^{2}\right) / \tilde{\Psi}^{-1}\left(1 / \nu_{\varphi, 2}(h)\right) \leq \Psi^{-1}(1 / h) / \Psi^{-1}\left(1 / \nu_{\varphi}(h)\right),
$$

since $\nu_{\varphi, 2}(h) \leq\left[\nu_{\varphi}(h)\right]^{2}$, where $\nu_{\varphi}(h)=\sup _{|w| \geq 1-h} N_{\varphi}(w)$.

In the opposite direction of Theorem 4.2 , one may ask if, for every Orlicz function $\Psi$, there exists a composition operator which is compact on $\mathfrak{B}^{\Psi}$ but not compact on $H^{\Psi}$. We showed in [7, Theorem 5.8, that this is the case for $\Psi(x)=\mathrm{e}^{x^{2}}-1$. Actually, this is always the case.

Proposition 4.4. For every Orlicz function $\Psi$ there exists a Blaschke product $B$ for which $C_{B}: \mathfrak{B}^{\Psi} \rightarrow \mathfrak{B}^{\Psi}$ is compact, whereas $C_{B}: H^{\Psi} \rightarrow H^{\Psi}$ is an isometry.

A weaker result is stated in [1], Theorem 13.

Proof. We proved in [10], Theorem 3.1, that for every function $\delta:(0,1) \rightarrow(0,1 / 2]$ there exists a Blaschke product $B$ such that $1-|B(z)| \geq \delta(1-|z|)$ for all $z \in \mathbb{D}$. We may replace $B(z)$ by $z B(z)$ without changing this almost inequality. Then $C_{B}: H^{\Psi} \rightarrow H^{\Psi}$ is an isometry since $B(0)=0$ and $|B(z)|=1$ for $|z|=1$ (see [15]). To prove the first assertion we shall use Theorem 3.2 with a suitable choice of $\delta$. Let

$$
F(t)=\frac{1}{2 \Psi\left(\left[\Psi^{-1}\left(1 / t^{2}\right)\right]^{2}\right)} .
$$

This is a non-decreasing function, and we take for $\delta$ Lévy's pseudo-inverse $G$ of $F$ defined by $G(s)=\inf \{t \geq 0 ; F(t) \geq s\}$. Then $\delta(1-|z|) \leq h$ if and only if $F(h) \geq 1-|z|$. Hence

$$
\begin{aligned}
\rho_{\varphi, 2}(h) & =\sup _{|\xi|=1} \mathcal{A}(\{z \in \mathbb{D} ; B(z) \in W(\xi, h)\}) \leq \mathcal{A}(\{z \in \mathbb{D} ; 1-|B(z)| \leq h\}) \\
& \leq \mathcal{A}(\{z \in \mathbb{D} ; \delta(1-|z|) \leq h\})=\mathcal{A}(\{z \in \mathbb{D} ; F(h) \geq 1-|z|\}) \\
& =1-[1-F(h)]^{2} \leq 2 F(h) .
\end{aligned}
$$


It follows that

$$
\frac{\Psi^{-1}\left(1 / h^{2}\right)}{\Psi^{-1}\left[1 / \rho_{\varphi, 2}(h)\right]} \leq \frac{\Psi^{-1}\left(1 / h^{2}\right)}{\Psi^{-1}[1 / 2 F(h)]}=\frac{\Psi^{-1}\left(1 / h^{2}\right)}{\left[\Psi^{-1}\left(1 / h^{2}\right)\right]^{2}}=\frac{1}{\Psi^{-1}\left(1 / h^{2}\right)} \underset{h \rightarrow 0}{\longrightarrow} 0,
$$

and that proves the compactness of $C_{B}: \mathfrak{B}^{\Psi} \rightarrow \mathfrak{B}^{\Psi}$.

\section{ACKNOWLEDGEMENT}

The fourth author was partially supported by a Spanish research project MTM2006-05622.

\section{REFERENCES}

[1] D. M. Boyd, Composition operators on the Bergman space, Colloq. Math. 34 (1975/76), no. 1, 127-136. MR0407644 (53:11416)

[2] C. C. Cowen and B. D. MacCluer, Composition Operators on Spaces of Analytic Functions, Studies in Advanced Mathematics, CRC Press, Boca Raton, FL (1995). MR1397026 (97i:47056)

[3] P. Duren and A. Schuster, Bergman Spaces, Mathematical Surveys and Monographs, 100, American Mathematical Society, Providence, RI (2004). MR2033762 (2005c:30053)

[4] W. H. Hastings, A Carleson measure theorem for Bergman spaces, Proc. Amer. Math. Soc. 52 (1975), 237-241. MR0374886 (51:11082)

[5] H. Hedenmalm, B. Korenblum, and K. Zhu, Theory of Bergman Spaces, Graduate Texts in Mathematics 199, Springer (2000). MR1758653 (2001c:46043)

[6] P. Lefèvre, D. Li, H. Queffélec, and L. Rodríguez-Piazza, A criterion of weak compactness for operators on subspaces of Orlicz spaces, Journal of Function Spaces and Applications, 6, No. 3 (2008), 277-292. MR2444526 (2009i:46058)

[7] P. Lefèvre, D. Li, H. Queffélec, and L. Rodríguez-Piazza, Composition operators on HardyOrlicz spaces, Memoirs Amer. Math. Soc. 207 (2010) No. 974. MR2681410 (2011m:47051)

[8] P. Lefèvre, D. Li, H. Queffélec, and L. Rodríguez-Piazza, Some examples of compact composition operators on $H^{2}$, J. Funct. Anal. 255, no. 11 (2008), 3098-3124. MR2464571 (2009j:47044)

[9] P. Lefèvre, D. Li, H. Queffélec, and L. Rodríguez-Piazza, Nevanlinna counting function and Carleson function of analytic maps, Math. Ann. 351 (2011), 305-326. MR2836660 (2012f:47065)

[10] P. Lefèvre, D. Li, H. Queffélec, and L. Rodríguez-Piazza, Some revisited results about composition operators on Hardy spaces, Revista Mat. Iberoamer. 28 (1) (2012), 57-76. MR2904130

[11] D. Li, Compact composition operators on Hardy-Orlicz and Bergman-Orlicz spaces, RACSAM 105 (2) (2011), 247-260. MR2826705(2012h:47056)

[12] D. Li and H. Queffélec, Introduction à l'étude des espaces de Banach. Analyse et probabilités, Cours Spécialisés 12, Société Mathématique de France, Paris (2004). MR2124356 (2006c:46012)

[13] D. Li, H. Queffélec, and L. Rodríguez-Piazza, Infinitesimal Carleson property for weighted measures induced by analytic self-maps of the unit disk, Complex Anal. Oper. Theory, to appear.

[14] B. MacCluer and J. Shapiro, Angular derivatives and compact composition operators on the Hardy and Bergman spaces, Canad. J. Math. 38, no. 4 (1986), 878-906. MR854144 (87h:47048)

[15] E. A. Nordgren, Composition operators, Canad. J. Math. 20 (1968), 442-449. MR.0223914 $(36: 6961)$

[16] Ch. Pommerenke, Boundary Behaviour of Conformal Maps, Grundlehren der Mathematischen Wissenschaften 299, Springer-Verlag, Berlin (1992). MR.1217706 (95b:30008)

[17] M. M. Rao and Z. D. Ren, Theory of Orlicz Spaces, Pure and Applied Mathematics 146, Marcel Dekker, Inc. (1991). MR 1113700 (92e:46059)

[18] J. H. Shapiro, The essential norm of a composition operator, Annals of Math. 125 (1987), 375-404. MR881273 (88c:47058)

[19] J. H. Shapiro and P. D. Taylor, Compact, nuclear, and Hilbert-Schmidt composition operators on $H^{2}$, Indiana Univ. Math. J. 23 (1973), 471-496. MR0326472 (48:4816) 
[20] K. Zhu, Operator Theory in Function Spaces, second edition, Mathematical Surveys and Monographs, Volume 138, American Mathematical Society (2007). MR2311536|(2008i:47064)

Laboratoire de Mathématiques de Lens EA 2462, Fédération CNRS Nord-Pas-deCalais FR 2956, Université Lille-Nord-De-France UArtois, F-62 300 Lens, France

E-mail address: pascal.lefevre@euler.univ-artois.fr

Laboratoire de Mathématiques de Lens EA 2462, Fédération CNRS Nord-Pas-deCalais FR 2956, Université Lille-Nord-De-France UArtois, Faculté des Sciences Jean Perrin, Rue Jean Souvraz, S.P. 18, F-62 300 Lens, France

E-mail address: daniel.li@euler.univ-artois.fr

Laboratoire Paul Painlevé U.M.R. CNRS 8524, Université Lille-Nord-De-France USTL, F-59655 Villeneuve D'AscQ Cedex, France

E-mail address: Herve.Queffelec@univ-lille1.fr

Facultad de Matemáticas, Departamento de Análisis Matemático \& imUS, Universidad de Sevilla, Apartado de Correos 1160, 41080 Sevilla, Spain

E-mail address: piazza@us.es 